Stochastic Systems

arXiv: arXiv: 1206.3390

\title{
STATE-INDEPENDENT IMPORTANCE SAMPLING FOR RANDOM WALKS WITH REGULARLY VARYING INCREMENTS
}

\author{
By Karthyek R. A. Murthy, ${ }^{*}$ Sandeep Juneja* And \\ Jose BlancheT ${ }^{\dagger}$ \\ Tata Institute of Fundamental Research ${ }^{*}$ and Columbia University ${ }^{\dagger}$
}

\begin{abstract}
We develop importance sampling based efficient simulation techniques for three commonly encountered rare event probabilities associated with random walks having i.i.d. regularly varying increments; namely, 1) the large deviation probabilities, 2) the level crossing probabilities, and 3) the level crossing probabilities within a regenerative cycle. Exponential twisting based state-independent methods, which are effective in efficiently estimating these probabilities for light-tailed increments are not applicable when the increments are heavy-tailed. To address the latter case, more complex and elegant state-dependent efficient simulation algorithms have been developed in the literature over the last few years. We propose that by suitably decomposing these rare event probabilities into a dominant and further residual components, simpler state-independent importance sampling algorithms can be devised for each component resulting in composite unbiased estimators with desirable efficiency properties. When the increments have infinite variance, there is an added complexity in estimating the level crossing probabilities as even the well known zero-variance measures have an infinite expected termination time. We adapt our algorithms so that this expectation is finite while the estimators remain strongly efficient. Numerically, the proposed estimators perform at least as well, and sometimes substantially better than the existing state-dependent estimators in the literature.
\end{abstract}

1. Introduction. In this paper, we develop importance sampling algorithms involving simple, state-independent changes of measure for the efficient estimation of large deviations, and level crossing probabilities of random walks with regularly varying increments. Specifically, let $X, X_{1}, X_{2}, \ldots$ denote a collection of independent and identically distributed (i.i.d.) random variables such that $\mathbb{P}\{X>x\}=L(x) x^{-\alpha}$, for some $\alpha>1$ and a slowly varying function ${ }^{1} L(\cdot)$. Note that $\alpha>2$ ensures finite variance for $X$ whereas

AMS 2000 subject classifications: Primary 60G50, 60J05, 68W40; secondary 60J20.

Keywords and phrases: State-independent importance sampling, rare-event simulation, heavy-tails, random walks, single-server queue, insurance ruin.

${ }^{1}$ That is, $\lim _{x \rightarrow \infty} L(t x) / L(x)=1$ for any $t>0$; see Section 2.3 for examples and more details 
$\alpha<2$ implies that $X$ has infinite variance. Set

$$
S_{0}=0, \quad S_{n}=X_{1}+\ldots+X_{n}, \text { and } M_{n}=\max _{k \leq n} S_{k}, \text { for } n \geq 1 .
$$

Further, let $M:=\sup _{n} S_{n}, \tau_{b}:=\inf \left\{n \geq 1: S_{n}>b\right\}$ and the regenerative cycle duration $\tau:=\inf \left\{n \geq 1: S_{n} \leq 0\right\}$. We are interested in importance sampling based efficient estimation of:

1) Large deviations probabilities $\mathbb{P}\left\{S_{n}-n \mathbb{E} X>b\right\}$ for $b>n^{\beta+\epsilon}$, with $\beta:=(\alpha \wedge 2)^{-1}$ and any fixed $\epsilon>0$, as $n \nearrow \infty$,

2) Level crossing probabilities $\mathbb{P}\left\{\tau_{b}<\infty\right\}$, or equivalently, the tail probabilities $\mathbb{P}\{M>b\}$, when $\mathbb{E} X<0$ and $b \nearrow \infty$,

3) Level crossing probabilities within the regenerative cycle $\mathbb{P}\left\{\tau_{b}<\tau\right\}$, or equivalently, the tail probabilities of regenerative cycle maximum $\mathbb{P}\left\{M_{\tau}>b\right\}$, when $\mathbb{E} X<0, \alpha>2$ and $b \nearrow \infty$.

Our methodology for estimating the large deviations probabilities easily extends to the efficient estimation of $\mathbb{P}\left\{S_{N}>u\right\}$ for random $N$, when $N$ is light-tailed ${ }^{2}$ and independent of increments $\left\{X_{n}\right\}$ (popular in literature are $N$ fixed or geometrically distributed) as $u \nearrow \infty$. However, in the interest of space, we do not explicitly consider the 'random sum tail probabilities' estimation problem in this paper.

Importance sampling via appropriate change of measure has been extremely successful in efficiently simulating rare events, and has been studied extensively in both the light and heavy tailed settings (see, e.g., [5] for an introduction to rare event simulation and applications). In importance sampling for random walks, state-dependence essentially means that the sampling distribution for generating the increment $X_{k}$ depends on the realized values of $X_{1}, \ldots, X_{k-1}$ (typically, through $S_{k-1}$ ); state-independence on the other hand implies that samples of $X_{1}, \ldots, X_{n}$ can be drawn independently. State-independent methods often enjoy advantages over statedependent ones in terms of complexity of generating samples and ease of implementation. The zero-variance changes of measure for estimating the large deviations and the level crossing probabilities are well known and are state-dependent (see, e.g., [32]). While typically unimplementable, they provide guidance in search for implementable approximately zero-variance importance sampling techniques.

In the light-tailed settings, large deviations analysis can be used to show that exponential twisting based state-independent importance sampling well

\footnotetext{
${ }^{2}$ As is well-known, $X$ is light-tailed if the moment generating function $\mathbb{E}[\exp (\theta X)]$ is finite for some $\theta>0$, and is heavy-tailed otherwise.
} 
approximates the zero-variance measures (see, e.g., [5]) for efficiently estimating the large deviations as well as the level crossing probabilities (see, e.g., [38] and [39]). However, development of state-independent techniques for these probabilities is considered harder in the heavy-tailed settings. In [4], Asmussen et al. provide an account of failure of simple large deviations based simulation methods that approximate zero-variance measure in heavy-tailed systems. Further, Bassamboo et al. [8] prove that any importance sampling change of measure that prescribes increments to be drawn in an i.i.d. fashion cannot efficiently estimate probabilities of level crossing within a regenerative cycle of a heavy-tailed random walk. The fact that the zero-variance measures for estimating both the large deviations and the level crossing probabilities are state-dependent, and the above mentioned negative results, have motivated research over the last few years in development of complex and elegant state-dependent algorithms to efficiently estimate these probabilities (see, e.g., $[22,10,13,14,18]$ ).

In this paper we introduce simple state-independent change of measures to estimate the large deviations and the level crossing probabilities with regularly varying increments. We show that the proposed methods are provably efficient $^{3}$ and perform at least as well as the existing state-dependent algorithms. Thus our key contribution is to question the prevailing view that one needs to resort to state-dependent methods for efficient computation of rare event probabilities involving 'large number' of heavy-tailed random variables. A key idea to be exploited in the estimation of probabilities considered is the fact that the corresponding rare event occurrence is governed by the "single big jump" principle, that is, the most likely paths leading to the occurrence of the rare event have one of the increments taking large value (see, for e.g., [26] and the references therein). Our approach for estimating the large deviations probability $\mathbb{P}\left\{S_{n}>b\right\}$ relies on decomposing it into a dominant and a residual component, and developing efficient estimation techniques for both. For estimating the level crossing probability $\mathbb{P}\left\{\tau_{b}<\infty\right\}$, in addition to such a decomposition, we partition the event of interest into several blocks that are sampled using appropriate randomization. When the increments $X_{n}$ have infinite variance, there is an added complexity in estimating the level crossing probabilities $\mathbb{P}\left\{\tau_{b}<\infty\right\}$ as even the well

\footnotetext{
${ }^{3}$ We show that the estimators have asymptotically vanishing relative error; this corresponds to their coefficient of variation converging to zero as the event becomes rarer. We also have a related weaker notion of strong efficiency where the coefficient of variation of the estimators, and subsequently the number of i.i.d. replications required, remains bounded as the event becomes rarer. Weak efficiency is another standard notion of performance in rare event simulation corresponding to a slow increase in the number of replications required as the event becomes rarer. These are briefly reviewed in Section 2.2.
} 
known zero-variance measure is known to have an infinite expected termination time. We modify our algorithms so that this expectation remains finite while the estimators remain strongly efficient although they may no longer have asymptotically vanishing relative error.

Our specific contributions are as follows:

1. We provide importance sampling estimators that achieve asymptotically vanishing relative error for the estimation of $\mathbb{P}\left\{S_{n}>b\right\}$, as $n \nearrow \infty$. Given $n$ and $\epsilon>0$, our simulation methodology is uniformly efficient for values of $b$ larger than $n^{\frac{1}{2}+\epsilon}$ when the increments $X_{n}$ have finite variance, and for $b>n^{\frac{1}{\alpha}+\epsilon}$ in the case of increments having infinite variance - thus operating throughout the large deviations regime where the well-known asymptotics $\mathbb{P}\left\{S_{n}>b\right\} \sim n \bar{F}(b)$ hold. Further, this is the first instance that we are aware of where efficient simulation techniques for the large deviations probability include the case of increments having infinite variance, which is not uncommon in practical applications involving heavy-tailed random variables.

2. For $\alpha>1$, we develop unbiased estimators for level crossing probabilities $\mathbb{P}\left\{\tau_{b}<\infty\right\}$ that achieve vanishing relative error as $b \nearrow \infty$. These estimators require an overall computational effort that scales as $O(b)$ when the variance of increments $X_{n}$ is finite. This is similar to the complexity of the zero variance operator since, as is well known, the latter requires order $\mathbb{E}\left[\tau_{b} \mid \tau_{b}<\infty\right]$ computation in generating a single sample and this is known to be linear in $b$ when the variance of increments is finite (see $[6]$ ). However, since $\mathbb{E}\left[\tau_{b} \mid \tau_{b}<\infty\right]=\infty$ for the case of increments having infinite variance, the zero-variance change of measure might not necessarily be a good benchmark, because from a computational standpoint any useful estimator needs to have finite expected termination time. For random walks with increments having infinite variance, we develop algorithms such that:

(a) When $\alpha>1.5$, the associated estimators are strongly efficient and have $O(b)$ expected termination time. As a converse, we also prove that for $\alpha<1.5$ no algorithm can be devised in our framework that has both the variance and expected termination time simultaneously finite. The situation is more nuanced when $\alpha=1.5$ and depends on the form of the slowly varying function $L(\cdot)$.

(b) When $\alpha \leq 1.5$, each replication of the estimator terminates in $O(b)$ time in expectation; also we require only $O(1)$ replications to achieve a given relative error, thus resulting in overall complexity of $O(b)$. 
The above results for infinite increment variance, and in particular the bottleneck arising at $\alpha=1.5$, closely mirror the results proved in [14] where vastly different state-dependent algorithms are considered.

3. Similarly, for the level crossing probabilities $\mathbb{P}\left\{\tau_{b}<\tau\right\}$, we partition the event into dominant and residual components, and devise changes of measure separately for the component events. The resulting importance sampling estimators are proved to be strongly efficient, as $b \nearrow \infty$. This is significant considering the negative result of [9] in context, where it is proved that no state-independent change of measure can be devised to efficiently simulate $\left\{\tau_{b}<\tau\right\}$. Our analysis thus informs that decomposing the event of interest in a suitable manner may be a reasonable way to address problems where designing importance sampling measures are known to be difficult.

A brief discussion on practical applications and a literature review may be in order: Efficient estimation of the level crossing probability is important in many practical contexts, e.g., in computing steady state probability of delays in $G I / G I / 1$ queues and in ruin probabilities in insurance settings (see, e.g., [5]). Siegmund [39] provides the first weakly efficient importance sampling algorithm for estimating the level crossing probabilities when the increments $X_{n}$ are light-tailed using large deviations based exponentially twisted change of measure. In [38], Sadowsky and Bucklew develop a weakly efficient algorithm for estimating $\mathbb{P}\left(S_{n}>n a\right)$ for $a>\mathbb{E} X$, and $X$ light-tailed, again using exponential twisting based importance sampling distribution (also see $[37,23,12,21,2]$ for related analysis). This problem is important mainly because it forms a building block to many more complex rare event problems involving combination of renewal processes: for examples in queueing, see [35] and in financial credit risk modeling, see [27] and [9].

Research on efficient simulation of rare events involving heavy-tailed variables first focussed on probabilities such as $\mathbb{P}\left\{S_{N}>b\right\}$ in the simpler asymptotic regime where $N$ is fixed or geometrically distributed and $b \nearrow \infty$. In this simpler setting state-independent algorithms are easily designed (see, e.g., $[4,31,7])$. In [34], it is shown that a variant capped exponential twisting based state-independent importance sampling, which does not involve any decomposition, provides a strongly efficient estimator for the large deviations probability that we consider in this paper.

Statistical analysis reveals that heavy-tailed distributions are very common in practice: in particular, heavy-tailed increments with infinite variance are a convenient means to explain the long-range dependence observed in tele-traffic data, and to model highly variable claim sizes in insurance settings. Popular references to this strand of literature include [24, 36, 1]. 
The organization of the remaining paper is as follows: In Section 2 we discuss preliminary concepts relevant to the problems addressed. We propose our importance sampling method for estimating the large deviations probability and prove its efficiency in Section 3. In Section 4, we develop algorithms for estimating the level crossing probabilities $\mathbb{P}\left\{\tau_{b}<\infty\right\}$. Proofs of some of the key results pertaining to efficiency and expected termination time of algorithms proposed in Section 4 are presented in Section 5. The efficient simulation of level crossing within a regenerative cycle is considered in Section 6. Numerical experiments supporting our algorithms are given in Section 7 followed by a brief conclusion in Section 8. Some of the more technical proofs are presented in the appendix.

2. Preliminary Background. In this section we briefly review the use of importance sampling in estimating rare event probabilities. We use Landau's notation for describing asymptotic behaviour of functions: for given functions $f: \mathbb{R}^{+} \rightarrow \mathbb{R}^{+}$and $g: \mathbb{R}^{+} \rightarrow \mathbb{R}^{+}$, we say $f(x)=O(g(x))$ if there exists $c_{1}>0$ and $x_{1}$ large enough such that $f(x) \leq c_{1} g(x)$ for all $x>x_{1}$; and $f(x)=\Omega(g(x))$ if there exists $c_{2}>0$ and $x_{2}$ large enough such that $f(x) \geq c_{2} g(x)$ for all $x>x_{2}$. We use $f(x)=o(g(x))$ if $f(x) / g(x) \rightarrow 0$, and $f(x) \sim g(x)$ if $f(x) / g(x) \rightarrow 1$, as $x \nearrow \infty$. Throughout this paper, if a probability measure is specified with a suffix, the expectation and variance operators evaluated with respect to that measure are specified with the same suffix. For example, $\mathbb{E}_{n}[\cdot]$ and $\operatorname{Var}_{n}[\cdot]$ denote expectation and variance operators associated with the measure $\mathbb{P}_{n}(\cdot)$.

2.1. Rare event simulation and importance sampling. Let $A$ denote a rare event on the probability space $(\Omega, \mathcal{F}, \mathbb{P})$, i.e., $z:=\mathbb{P}(A)>0$ is small (in our setup $A$ corresponds to the events $\left\{S_{n}>b\right\}$ or $\left.\left\{\tau_{b}<\infty\right\}\right)$. Suppose that we are interested in obtaining an estimator $\hat{z}$ for $z$ such that the relative error $|\hat{z}-z| / z$ is not more than $\epsilon$, with probability at least $1-\delta$, for given $\epsilon$ and $\delta>$ 0 . Naive simulation for estimating $z$ involves drawing $N$ independent samples of the indicator $\mathbb{I}(A)$ and taking their sample mean as the estimator. For a different measure $\mathbb{P}_{1}(\cdot)$ such that the Radon-Nikodym derivative $d \mathbb{P} / d \mathbb{P}_{1}$ is well defined on $A$, we have:

$$
\mathbb{P}(A)=\int_{A} \frac{d \mathbb{P}}{d \mathbb{P}_{1}}(\omega) d \mathbb{P}_{1}(\omega)=\mathbb{E}_{1}\left[L \mathbb{I}_{A}\right]
$$

where $L:=d \mathbb{P} / d \mathbb{P}_{1}$ and $\mathbb{E}_{1}[\cdot]$ is the expectation associated with $\mathbb{P}_{1}(\cdot)$. Define $Z:=L \mathbb{I}(A)$; then $Z$ is an unbiased estimator of $z$ under measure $\mathbb{P}_{1}(\cdot)$. If $N$ i.i.d samples $Z_{1}, \ldots, Z_{N}$ of $Z$ are drawn from $\mathbb{P}_{1}(\cdot)$, then by the strong law 
of large numbers we have:

$$
\hat{z}_{N}:=\frac{Z_{1}+\ldots+Z_{N}}{N} \rightarrow z \text { a.s. }
$$

as $N \nearrow \infty$. This method of arriving at an estimator is called importance sampling (IS). The measure $\mathbb{P}_{1}(\cdot)$ is called the importance sampling measure and $Z$ is called an importance sampling estimator. Using Chebyshev's inequality allows us to find an upper bound on the number of replications $N$ required to achieve the desired relative precision:

$$
\mathbb{P}\left(\frac{\left|\hat{z}_{N}-z\right|}{z}>\epsilon\right) \leq \frac{\operatorname{Var}_{1}\left[\hat{z}_{N}\right]}{z^{2} \epsilon^{2}}=\frac{C V^{2}(Z)}{N \epsilon^{2}} .
$$

Here $\operatorname{Var}_{1}[\cdot]:=\mathbb{E}_{1}\left[(\cdot)^{2}\right]-\mathbb{E}_{1}[\cdot]^{2}$ is the variance operator associated with measure $\mathbb{P}_{1}(\cdot)$ and $C V(Z)=\sqrt{\operatorname{Var}_{1}[Z]} / z$ is the coefficient of variation of $Z$. This enables us to conclude that if we generate at least

$$
N=\frac{C V^{2}(Z)}{\delta \epsilon^{2}}
$$

i.i.d. samples of $Z$ for computing $\hat{z}_{N}$, we can guarantee the desired relative precision. In naive simulation we use the measure $\mathbb{P}(\cdot)$ itself and have $Z=\mathbb{I}(A)$ as the estimator; so the number of samples required in (1) grows (roughly proportional to $z^{-1}$ ) to infinity if $z \searrow 0$. As is well known, the choice $\mathbb{P}^{*}(\cdot):=\mathbb{P}(\cdot \mid A)$ as an importance sampling measure yields zero variance for the associated estimator $Z=z \mathbb{I}(A)$ (see e.g., [5]); then every sample obtained in simulation equals $z$ with $\mathbb{P}^{*}(\cdot)$ probability 1 . However, the explicit dependence of $Z$ on $z$, the quantity which we want to estimate, makes this method impractical.

2.2. Efficiency notions of algorithms. Consider a family of events $\left\{A_{n}\right.$ : $n \geq 1\}$ such that $z_{n}:=\mathbb{P}\left(A_{n}\right) \searrow 0$ as the rarity parameter $n \nearrow \infty$. For an importance sampling algorithm to compute $\left(z_{n}: n \geq 1\right)$, we come up with a sequence of changes of measure $\left(\mathbb{P}_{n}(\cdot): n \geq 1\right)$ and estimators $\left(Z_{n}: n \geq 1\right)$ such that $\mathbb{E}_{n} Z_{n}=z_{n}$.

DeFinition 1. The sequence $\left(Z_{n}: n \geq 1\right)$ of unbiased importance sampling estimators of $\left\{z_{n}: n \geq 1\right\}$, is said to achieve asymptotically vanishing relative error if,

$$
\varlimsup_{n \rightarrow \infty} \frac{\operatorname{Var}_{n}\left[Z_{n}\right]}{z_{n}^{2}}=0
$$


The sequence $\left(Z_{n}: n \geq 1\right)$ is said to be strongly efficient if,

$$
\sup _{n} \frac{\operatorname{Var}_{n}\left[Z_{n}\right]}{z_{n}^{2}}<\infty
$$

and weakly efficient if for all $\epsilon>0$,

$$
\varlimsup_{n \rightarrow \infty} \frac{\operatorname{Var}_{n}\left[Z_{n}\right]}{z_{n}^{2-\epsilon}}=0
$$

The significance of these definitions can be seen from (1): if an algorithm is strongly efficient, the number of simulation runs required to guarantee the desired relative precision stays bounded as $n \nearrow \infty$. If $\operatorname{Var}\left(Z_{n}\right)=o\left(z_{n}^{2}\right)$, then $\left(Z_{n}: n \geq 1\right)$ satisfies asymptotically vanishing relative error property. As a result, it is enough to generate $o\left(\delta^{-1} \epsilon^{-2}\right)$ i.i.d. replications of the estimator. As is apparent from the definition, all strongly efficient algorithms are weakly efficient, and vanishing relative error is the strongest notion among all three. Also it can be verified that naive simulation is not even weakly efficient.

2.3. Regularly varying tails. A function $L: \mathbb{R}^{+} \rightarrow \mathbb{R}^{+}$is said to be slowly varying at infinity if

$$
\lim _{x \rightarrow \infty} \frac{L(t x)}{L(x)}=1, \text { for all } t>0 .
$$

Some examples of slowly varying functions include $|\log x|^{\beta}$ for any $\beta \in \mathbb{R}, 1-$ $e^{-x}$, etc. A random variable $X$ is said to be regularly varying with index $-\alpha$ if for each $t>0$,

$$
\lim _{x \rightarrow \infty} \frac{\mathbb{P}\{X>t x\}}{\mathbb{P}\{X>x\}}=t^{-\alpha} .
$$

In other words, $\mathbb{P}\{X>x\}=x^{-\alpha} L(x)$ for some slowly varying function $L(\cdot)$. It can be easily verified that any regularly varying random variable $X$ is heavy-tailed: that is, $\mathbb{E}[\exp (\theta X)]=\infty$ for any $\theta>0$. These regularly varying distribution functions capture the concept of polynomially decaying tails, and form an important class of heavy-tailed distributions. The following properties of regularly varying functions will be useful in our analysis:

1) Karamata's theorem: For any regularly varying function $V(\cdot)$ with in$\operatorname{dex}-\alpha$, if $\beta$ is such that $\alpha-\beta>1$, then

$$
\int_{x}^{\infty} u^{\beta} V(u) d u \sim \frac{x^{\beta+1} V(x)}{\alpha-\beta-1}, \text { as } x \quad \nearrow \infty .
$$


This result, a part of Karamata's theorem (cf. Theorem 1 in Chapter VIII.9 of [25]), provides an asymptotic characterization of integrated tails.

2) Potter's bounds: Potter's bounds: If $L(\cdot)$ is a slowly varying function, then as in Theorem 1.1.4 of [16], for any $\delta>0$, there exists a $t_{\delta}>0$ such that for all $t$ and $v$ satisfying $t \geq t_{\delta}$ and $v t \geq t_{\delta}$,

$$
(1-\delta) \min \left\{v^{\delta}, v^{-\delta}\right\} \leq \frac{L(v t)}{L(t)} \leq(1+\delta) \max \left\{v^{\delta}, v^{-\delta}\right\}
$$

3. Simulation of $\left\{S_{n}>\boldsymbol{b}\right\}$. Let $X$ be a zero mean random variable with distribution $F(\cdot)$ satisfying the following:

Assumption 1. The tail probabilities of $X$ are given by $\bar{F}(x):=\mathbb{P}\{X>$ $x\}=x^{-\alpha} L(x)$, for some slowly varying function $L(\cdot)$ and $\alpha>1$. Further if $\operatorname{Var}[X]=\infty$, the tail probabilities of $X$ satisfy the following condition:

$$
\varlimsup_{x \rightarrow \infty} \frac{\mathbb{P}\{X<-x\}}{\mathbb{P}\{X>x\}}<\infty
$$

For the independent collection $\left(X_{n}: n \geq 1\right)$ of random variables which are distributed identically as $X$, define the random walk $\left(S_{n}: n \geq 0\right)$ as below:

$$
S_{0}=0, \text { and } S_{n}=X_{1}+\ldots+X_{n} \text { for } n \geq 1 \text {. }
$$

In this section we devise a simulation procedure for estimating the large deviation probabilities $\mathbb{P}\left\{S_{n}>b\right\}$ and prove its efficiency as $n \nearrow \infty$. For accomplishing this, we quickly review the following well-known asymptotics: Let $\beta:=(\alpha \wedge 2)^{-1}$. When $\mathbb{E} X^{2}<\infty$, we have that

$$
\frac{n \log n}{b^{2}} \int_{|x| \leq b} x^{2} F(d x) \leq \frac{n \log n}{b^{2}} \mathbb{E} X^{2} \searrow 0 \text {, as } n \nearrow \infty
$$

uniformly for $b>n^{\frac{1}{2}+\epsilon}$. Similarly when $\mathbb{E} X^{2}=\infty$, since $\mathbb{E} X=0$, it follows 
from Assumption 1 that

$$
\begin{aligned}
\frac{n}{b} \int_{|x| \leq b} x F(d x) & =-\frac{n}{b} \int_{|x|>b} x F(d x) \\
& =\frac{n}{b}\left(\int_{x<-b}|x| F(d x)+\int_{x>b}|x| \bar{F}(d x)\right) \\
& \leq \frac{2 n}{b}\left(\int_{x>b} x \bar{F}(d x)\right)(1+o(1)) \\
& =\frac{2 n}{b}\left(b \bar{F}(b)+\int_{b}^{\infty} \bar{F}(u) d u\right)(1+o(1)) \\
& =\frac{2 n}{b}\left(b \bar{F}(b)+\frac{b \bar{F}(b)}{\alpha-1}\right)(1+o(1)) \searrow 0, \text { as } n \nearrow \infty
\end{aligned}
$$

uniformly for $b>n^{\beta+\epsilon}$. Then it follows from Theorem 3.3 of [19] that for any $\epsilon>0$,

$$
\sup _{b>n^{\beta+\epsilon}}\left|\frac{\mathbb{P}\left\{S_{n}>b\right\}}{n \bar{F}(b)}-1\right|=o(1), \text { as } n \nearrow \infty .
$$

A simple application of Bonferroni inequalities will yield

$$
\mathbb{P}\left\{\max \left\{X_{1}, \ldots, X_{n}\right\}>b\right\}=n \bar{F}(b)\left(1-\frac{1}{2} \bar{F}(b)+\frac{\theta}{6}(n \bar{F}(b))^{2}\right),
$$

for some $\theta$ in $(0,1)$. This indicates that the tail asymptotics of maximum and the sum of increments $\left\{X_{1}, \ldots, X_{n}\right\}$ match asymptotically.

The strategy for simulation is to partition the event $\left\{S_{n}>b\right\}$ based on whether the maximum of the increments $\left\{X_{1}, \ldots, X_{n}\right\}$ has exceeded the large value $b$ or not:

$$
\begin{aligned}
A_{\mathrm{dom}}(n, b) & :=\left\{S_{n}>b, \max _{k \leq n} X_{k} \geq b\right\} \text { and } \\
A_{\mathrm{res}}(n, b) & :=\left\{S_{n}>b, \max _{k \leq n} X_{k}<b\right\} .
\end{aligned}
$$

Such a partition is considered in [30] for the simulation of $\left\{S_{n}>b\right\}$ when $n$ is fixed. We prove the following result in the appendix:

Proposition 1. Under Assumption 1, given any $\epsilon>0$,

$$
\sup _{b>n^{\beta+\epsilon}} \frac{\mathbb{P}\left(A_{\mathrm{res}}(n, b)\right)}{n \bar{F}(b)}=o(1) \text { as } n \nearrow \infty \text {. }
$$


Therefore, the probability of the event $A_{\text {res }}$ is vanishingly small compared to the probability of $A_{\mathrm{dom}}$ as $n \nearrow \infty$; the suffixes stand to indicate that $A_{\text {dom }}$ is the dominant way of occurrence of $\left\{S_{n}>b\right\}$ for large $n$, and $A_{\text {res }}$ has only residual contributions. We estimate $\mathbb{P}\left(A_{\text {dom }}\right)$ and $\mathbb{P}\left(A_{\text {res }}\right)$ independently via different changes of measure that typify the way in which the respective events occur, and add the individual estimates to arrive at the final estimator for $\mathbb{P}\left\{S_{n}>b\right\}$.

3.1. Simulating $A_{\mathrm{dom}}$. For the simulation of $A_{\mathrm{dom}}$, we follow the two-step procedure outlined in [18]:

1. Choose an index $I$ uniformly at random from $\{1, \ldots, n\}$

2. For $k=1, \ldots, n$, generate a realization of $X_{k}$ from $F\left(\cdot \mid X_{k} \geq b\right)$ if $k=I$; otherwise, generate $X_{k}$ from $F(\cdot)$

Let $\mathbb{P}_{1}(\cdot)$ denote the measure induced when the increments are generated according to the above procedure; for brevity, we have chosen not to highlight the dependence of the importance sampling change of measure $\mathbb{P}_{1}(\cdot)$ on $n$ and $b$ in the notation. Note that the probability measure $\mathbb{P}(\cdot)$ is absolutely continuous with respect to $\mathbb{P}_{1}(\cdot)$ when restricted to $A_{\text {dom }}$. We have,

$$
d \mathbb{P}_{1}\left(x_{1}, \ldots, x_{n}\right)=\sum_{k=1}^{n} \frac{1}{n} \cdot \frac{d F\left(x_{1}\right) \ldots d F\left(x_{n}\right)}{\bar{F}(b)} \mathbf{1}\left(x_{k} \geq b\right) .
$$

Therefore the likelihood ratio on the set $A_{\text {dom }}$ is given by,

$$
\frac{d \mathbb{P}}{d \mathbb{P}_{1}}\left(X_{1}, \ldots, X_{n}\right)=\frac{n \bar{F}(b)}{\#\left\{X_{i} \geq b: 1 \leq i \leq n\right\}}
$$

and the resulting unbiased estimator for the evaluation of $\mathbb{P}\left(A_{\mathrm{dom}}\right)$ is,

$$
Z_{\mathrm{dom}}(n, b):=\frac{n \bar{F}(b)}{\#\left\{X_{i} \geq b: 1 \leq i \leq n\right\}} \mathbb{I}\left(A_{\mathrm{dom}}\right) .
$$

Generate $N$ independent realizations of $Z_{\text {dom }}$ and take their sample mean as an estimator of $\mathbb{P}\left(A_{\mathrm{dom}}\right)$. To evaluate how large $N$ should be chosen so that the computed estimate satisfies the given relative error specification, we need to obtain bounds on the variance of $Z_{\mathrm{dom}}$. Since $\#\left\{X_{i} \geq b: 1 \leq i \leq n\right\}$ is at least 1 , when the increments are drawn following the measure $\mathbb{P}_{1}(\cdot)$, we have: $Z_{\mathrm{dom}}(n, b) \leq n \bar{F}(b)$, and hence,

$$
\mathbb{E}_{1}\left[Z_{\text {dom }}^{2}(n, b)\right] \leq(n \bar{F}(b))^{2} .
$$


Also $\mathbb{E}_{1}\left[Z_{\text {dom }}(n, b)\right]=\mathbb{P}\left(A_{\text {dom }}(n, b)\right) \sim \mathbb{P}\left\{S_{n}>b\right\} \sim n \bar{F}(b)$, as $n \nearrow \infty$. Therefore we get,

$$
\operatorname{Var}_{1}\left[Z_{\mathrm{dom}}(n, b)\right]=o\left((n \bar{F}(b))^{2}\right) \text {, as } n \nearrow \infty .
$$

REMARK 1. Since $\mathbb{P}\left\{S_{n}>b, M_{n}>b\right\}=n \mathbb{P}\left\{S_{n}>b, M_{n}>b, M_{n}=X_{1}\right\}$, one can estimate $\mathbb{P}\left\{S_{n}>b, M_{n}>b, M_{n}=X_{1}\right\}$ efficiently by simulating $X_{1}$ from $F\left(\cdot \mid X_{1}>b\right)$ and the other increments from $F(\cdot)$. This avoids the simulation of an additional random variable $I$. However, we have presented the two step procedure above so that the simulation procedures introduced later in the paper appear intuitive.

REMARK 2. If the increments $X_{1}, \ldots, X_{n}$ are not identically distributed, and if at least one of the increments is regularly varying, then it can be verified that the following modification to the simulation of auxiliary random variable $I$ would suffice: Say $X_{j} \sim F_{j}(\cdot)$. Then choose $I=i$ from $\{1, \ldots, n\}$ with probability $\bar{F}_{i}(b) / \sum_{j=1}^{n} \bar{F}_{j}(b)$.

3.2. Simulating $A_{\text {res. }}$. We see that all the increments $\left\{X_{1}, \ldots, X_{n}\right\}$ are bounded from above by $b$ on the occurrence of event $A_{\text {res }}$. Though the bound on the increments vary with $n$, we can employ methods similar to exponential twisting of light-tailed random walks to simulate the event $A_{\text {res }}$, as illustrated in this section. For given $b$, define

$$
\Lambda_{b}(\theta):=\log \left(\int_{-\infty}^{b} \exp (\theta x) F(d x)\right), \quad \theta \geq 0 .
$$

Since the upper limit of integration is $b, \Lambda_{b}(\cdot)$ is well-defined for any positive value of $\theta$. For given values of $n$ and $b$, consider the distribution function $F_{\theta}(\cdot)$ satisfying,

$$
\frac{d F_{\theta}(x)}{d F(x)}=\exp \left(\theta_{n, b} x-\Lambda_{b}\left(\theta_{n, b}\right)\right) \mathbf{1}(x<b),
$$

for all $x \in \mathbb{R}$ and some $\theta_{n, b}>0$. Now the prescribed procedure is to just obtain independent samples of the increments $\left\{X_{1}, \ldots, X_{n}\right\}$ from $F_{\theta}(\cdot)$ and adjust via the likelihood ratio resulting due to the procedure of sampling from a different distribution $F_{\theta}(\cdot)$.

Let $\mathbb{P}_{2}(\cdot)$ denote the measure induced by sampling increments i.i.d from $F_{\theta}(\cdot)$. As before, for brevity, we have chosen not to highlight the dependence on parameters $n$ and $b$ in the notations $F_{\theta}(\cdot)$ and $\mathbb{P}_{2}(\cdot)$. For given values of 
$n$ and $b$, we have the following unbiased estimator for the computation of $\mathbb{P}\left(A_{\text {res }}\right)$ :

$$
Z_{\mathrm{res}}(n, b):=\exp \left(-\theta_{n, b} S_{n}+n \Lambda_{b}\left(\theta_{n, b}\right)\right) \mathbb{I}\left(A_{\text {res }}\right) .
$$

Now generate independent replications of $Z_{\text {res }}$ and take their sample mean as the computed estimate for $\mathbb{P}\left(A_{\text {res }}\right)$. However it remains to choose $\theta_{n, b}$. Since $S_{n}$ is larger than $b$ on $A_{\text {res }}$,

$$
Z_{\text {res }}(n, b) \leq \exp \left(-\theta_{n, b} b+n \Lambda_{b}\left(\theta_{n, b}\right)\right) \mathbb{I}\left(A_{\text {res }}\right) .
$$

If we choose

$$
\begin{aligned}
\theta_{n, b} & :=-\frac{\log (n \bar{F}(b))}{b}, \text { then } \\
Z_{\text {res }}(n, b) & \leq n \bar{F}(b) \exp \left(n \Lambda_{b}\left(\theta_{n, b}\right)\right) \mathbb{I}\left(A_{\text {res }}\right) .
\end{aligned}
$$

We use Lemma 1, which is proved in the appendix, to obtain an upper bound on the second moment of the estimator $Z_{\text {res }}$.

Lemma 1. Under Assumption 1, for the choice of $\theta_{n, b}$ as in (12),

$$
\exp \left(\Lambda_{b}\left(\theta_{n, b}\right)\right) \leq 1+\frac{1}{n}(1+o(1))
$$

as $n \nearrow \infty$, uniformly for $b>n^{\beta+\epsilon}$.

Therefore there exists a constant $c$ such that

$$
\exp \left(n \Lambda_{b}\left(\theta_{n, b}\right)\right) \leq c
$$

for all admissible values of $n$ and $b$. We evaluate the second moment of the estimator $Z_{\text {res }}$ through the equivalent expectation operation corresponding to the original measure $\mathbb{P}(\cdot)$ as below:

$$
\mathbb{E}_{2}\left[Z_{\mathrm{res}}^{2}(n, b)\right]=\mathbb{E}\left[Z_{\mathrm{res}}(n, b)\right] \leq c n \bar{F}(b) \mathbb{P}\left(A_{\text {res }}\right),
$$

where the last inequality follows from (13) and Lemma 1. From Proposition 1 , we have that $\mathbb{P}\left(A_{\text {res }}\right)=o(n \bar{F}(b))$. Therefore,

$$
\operatorname{Var}_{2}\left[Z_{\mathrm{res}}(n, b)\right]=o\left((n \bar{F}(b))^{2}\right), \text { as } n \nearrow \infty,
$$

thus arriving at the following theorem: 
THEOREM 1. If the realizations of the estimators $Z_{\mathrm{dom}}$ and $Z_{\mathrm{res}}$ are generated respectively from the measures $\mathbb{P}_{1}(\cdot)$ and $\mathbb{P}_{2}(\cdot)$, and if we let

$$
Z(n, b):=Z_{\mathrm{dom}}(n, b)+Z_{\mathrm{res}}(n, b),
$$

then under Assumption 1, the family of estimators $(Z(n, b): n \geq 1, b>$ $\left.n^{\beta+\epsilon}\right)$ achieves asymptotically vanishing relative error for the estimation of $\mathbb{P}\left\{S_{n}>b\right\}$, as $n \nearrow \infty$; that is,

$$
\frac{\operatorname{Var}_{n, b}[Z(n, b)]}{\mathbb{P}\left\{S_{n}>b\right\}^{2}}=o(1)
$$

as $n \nearrow \infty$, uniformly for $b>n^{\beta+\epsilon}$.

Here $\operatorname{Var}_{n, b}[\cdot]$ denotes the variance operator resulting due to the composite procedure of drawing realizations of $Z_{\text {dom }}$ and $Z_{\text {res }}$ from the measures $\mathbb{P}_{1}(\cdot)$ and $\mathbb{P}_{2}(\cdot)$ respectively.

Proof. Since the realizations of $Z_{\text {dom }}$ and $Z_{\text {res }}$ are obtained independent of each other, the variance of $Z$ is just the sum of variances of $Z_{\text {dom }}$ and $Z_{\text {res }}$ computed according to the measures from which they are generated; the proof is now evident from (10), (14) and (7).

REMARK 3. A consequence of the above theorem is that, due to (1), the number of i.i.d. replications of $Z(n, b)$ required to achieve $\epsilon$-relative precision with probability at least $1-\delta$ is at most $o\left(\epsilon^{-2} \delta^{-1}\right)$, which is independent of the rarity parameters $n$ and $b$. In our algorithm each replication demands $O(n)$ computational effort, thus requiring an overall computational cost of $O(n)$, as $n \nearrow \infty$.

REMARK 4. One can easily check that, this same simulation procedure can also be used to efficiently compute probabilities $\mathbb{P}\left\{S_{N}>b\right\}$ when $N$ is a light-tailed random variable independent of the increments $X_{n}$.

4. Simulation Methodology for $\left\{\tau_{b}<\infty\right\}$. As before, the sequence $\left(S_{n}: n \geq 0\right)$ with $S_{0}:=0$ and $S_{n}:=X_{1}+\ldots+X_{n}$ represents the random walk associated with the i.i.d collection $\left(X_{n}: n \geq 1\right)$. We have $\mathbb{E} X_{n}=0$, and $\mathbb{P}\left\{X_{n}>x\right\}=x^{-\alpha} L(x)$ for some slowly varying function $L(\cdot)$ and $\alpha>1$. Given $\mu>0$, let

$$
M:=\sup _{n}\left(S_{n}-n \mu\right) .
$$

Since $\left(S_{n}-n \mu: n \geq 0\right)$ is a random walk with negative drift, the random variable $M$ is proper. For $b>0$, recall that the first-passage time $\tau_{b}$ is defined 
as $\tau_{b}:=\inf \left\{n \geq 0: S_{n}-n \mu>b\right\}$. In this section we present simulation methods for the efficient computation of

$$
\mathbb{P}\{M>b\}=\mathbb{P}\left\{\tau_{b}<\infty\right\}, \text { as } b \nearrow \infty .
$$

Naive simulation of $\left\{\tau_{b}<\infty\right\}$ will require generation of all the increments until the partial sum $S_{n}-n \mu$ exceed $b$. Due to the negative drift of the random walk $\left(S_{n}-n \mu: n \geq 0\right)$, we have $\tau_{b} \nearrow \infty$ a.s. as $b \nearrow \infty$, and hence this method is not computationally feasible. To counter the prospect of generating uncontrollably large number of increment random variables in simulation, we re-express $\mathbb{P}\left\{\tau_{b}<\infty\right\}$ as below: Consider a strictly increasing sequence of integers $\left(n_{k}: k \geq 0\right)$ with $n_{0}=0$; also fix $p:=\left(p_{k}: k \geq 1\right)$ satisfying $p_{k}>0$ for all $k$ and $\sum_{k} p_{k}=1$; the vector $p$ can be seen as a probability mass function on positive integers. Consider an auxiliary random variable $K$ which takes the value of positive integer $k$ with probability $p_{k}$. Then

$$
\begin{aligned}
\mathbb{P}\left\{\tau_{b}<\infty\right\} & =\sum_{k \geq 1} p_{k} \frac{\mathbb{P}\left\{n_{k-1}<\tau_{b} \leq n_{k}\right\}}{p_{k}} \\
& =\mathbb{E}\left[\mathbb{E}\left[\frac{\mathbb{P}\left\{n_{K-1}<\tau_{b} \leq n_{K}\right\}}{p_{K}} \mid K\right]\right] .
\end{aligned}
$$

Now in a simulation run, if the realized value of the auxiliary random variable $K$ is $k$, generate a sample from a probability measure, possibly different from $\mathbb{P}(\cdot)$, of a random variable $Z_{k}$ that has $\mathbb{P}\left\{n_{k-1}<\tau_{b} \leq n_{k}\right\}$ as its expectation under the changed measure. Then equation (15) assures that taking the sample mean of i.i.d. replications of $Z_{K} / p_{K}$ following the changes of measure (to be explained in Section 4.2) for the generation of $\left\{Z_{k}: k \geq 1\right\}$ will yield an unbiased estimator for the quantity $\mathbb{P}\left\{\tau_{b}<\infty\right\}$.

The performance of any importance sampling algorithm following the outlined procedure will depend crucially on the choice of probabilities $p_{k}$, and the changes of measure employed to estimate $\mathbb{P}\left\{n_{k-1}<\tau_{b} \leq n_{k}\right\}$, for $k \geq 1$. The sequence $\left(n_{k}: k \geq 0\right)$ partitions non-negative integers into 'blocks' $\left(\left(n_{k-1}, n_{k}\right]: k \geq 1\right)$. For reasons that will be clear later, we choose the blocks $\left(n_{k-1}, n_{k}\right]$ in the following manner: Fix a positive integer $r>1$ and let,

$$
n_{0}=0, n_{k}=r^{k}, \text { for } k \geq 1 .
$$

In the following section, we present related asymptotics that will be useful in the efficiency analysis of the algorithms that are developed. 
4.1. Related Asymptotics. Recall that

$$
\tau_{b}:=\inf \left\{k: S_{k}>b+k \mu\right\} \text { and } M:=\sup _{n}\left(S_{n}-n \mu\right)
$$

The events $\{M>b\}$ and $\left\{\tau_{b}<\infty\right\}$ are the same. Let $\bar{F}_{I}(x):=\int_{x}^{\infty} \bar{F}(u) d u$ denote the integrated tail of $\bar{F}(\cdot)$. Under Assumption 1, it is well known (see, for example, [40]) that,

$$
\mathbb{P}\left\{\tau_{b}<\infty\right\} \sim \frac{1}{\mu} \bar{F}_{I}(b), \text { as } b \nearrow \infty .
$$

The asymptotics (16) hold for level crossing probabilities of random walks under more general increment distributions (see, for example, [33]).

The following finite-horizon asymptotics are also available if we make this non-restrictive smoothness assumption on the tail probabilities $\bar{F}(\cdot)$ :

Assumption 2. There exists a $t_{0}>0$ such that the slowly varying function $L(\cdot)$ in $\bar{F}(x)=x^{-\alpha} L(x)$ is continuously differentiable for all $t \geq t_{0}$. Further $L(\cdot)$ satisfies,

$$
L^{\prime}(x)=o\left(\frac{L(x)}{x}\right) \text {, as } x \nearrow \infty
$$

If $X$ is such that $\operatorname{Var}[X]<\infty$ and it satisfies Assumptions 1 and 2, then from Theorem 6 of [15], we have uniformly in $n$ that,

$$
\mathbb{P}\left\{\tau_{b} \leq n\right\}=\left(\sum_{j=1}^{n} \bar{F}(b+j \mu)\right)\left(1+O\left(\frac{1}{b}\right)\right)+o(\sqrt{b \wedge n} \bar{F}(b)) .
$$

When $\operatorname{Var}[X]=\infty$, under Assumptions 1 and 2, it follows from Theorem 2.4 of [17] that uniformly for all $n, b$ satisfying $n \bar{F}(b)=o(1)$,

$$
\mathbb{P}\left\{\tau_{b} \leq n\right\}=\left(\sum_{j=1}^{n} \bar{F}(b+j \mu)\right)\left(1+O\left(\frac{n^{\frac{1}{\alpha}+\epsilon}}{b}\right)\right)
$$

for every $\epsilon>0$.

The following characterization of the zero-variance measure $\mathbb{P}\left\{\cdot \mid \tau_{b}<\infty\right\}$ (see Theorem 1.1 of [6]) sheds light on how the first passage over a level $b$ 
happens asymptotically: If we use $a(b):=\bar{F}_{I}(b) / \bar{F}(b)$, then conditional on $\tau_{b}<\infty$,

$$
\left(\frac{\tau_{b}}{a(b)},\left(\frac{S_{\left\lfloor u \tau_{b}\right\rfloor}}{\tau_{b}}: 0 \leq u<1\right), \frac{S_{\tau_{b}}-b}{a(b)}\right) \Longrightarrow\left(\frac{Y_{0}}{\mu},(-u \mu: 0 \leq u<1), Y_{1}\right)
$$

in $\mathbb{R} \times D[0,1) \times \mathbb{R}$. The joint law of $Y_{0}, Y_{1}$ is defined as follows: for $y_{0}, y_{1} \geq$ $0, \mathbb{P}\left\{Y_{0}>y_{0}, Y_{1}>y_{1}\right\}=\mathbb{P}\left\{Y_{1}>y_{0}+y_{1}\right\}$ with $Y_{0} \stackrel{d}{=} Y_{1}$, and

$$
\mathbb{P}\left\{Y_{1}>y_{1}\right\}=\frac{1}{\left(1+y_{1} /(\alpha-1)\right)^{\alpha-1}} \text {. }
$$

4.2. Efficient simulation of $\left\{n_{k-1}<\tau_{b} \leq n_{k}\right\}$. In this section we identify importance sampling changes of measure for the efficient computation of the probabilities $\mathbb{P}\left\{n_{k-1}<\tau_{b} \leq n_{k}\right\}$. Define the following events:

$$
A_{k}=\bigcup_{i=n_{k-1}+1}^{n_{k}}\left\{X_{i}>b+i \mu\right\} \text { and } B_{k}=\bigcap_{i=1}^{n_{k}}\left\{X_{i}<b+n_{k-1} \mu\right\} \text {. }
$$

The events $A_{k}$ and $B_{k}$ are defined in the same spirit as that of $A_{\text {dom }}$ and $A_{\text {res }}$ in the simulation of $\left\{S_{n}>b\right\}$ in Section 3: the event $A_{k}$ includes sample paths that have at least one "big" jump of appropriate size in one of the increments indexed between $n_{k-1}$ and $n_{k}$, whereas on the other set $B_{k}$, we have all the increments bounded from above. As in the simulation of large deviation probabilities of sums of random variables in Section 3, we can partition the event $\left\{n_{k-1}<\tau_{b} \leq n_{k}\right\}$ into:

$\left\{n_{k-1}<\tau_{b} \leq n_{k}, A_{k}\right\},\left\{n_{k-1}<\tau_{b} \leq n_{k}, B_{k}\right\}$ and $\left\{n_{k-1}<\tau_{b} \leq n_{k}, \bar{A}_{k} \cap \bar{B}_{k}\right\}$,

and arrive at unbiased estimators for their probabilities separately via different importance sampling measures. Here $\bar{A}$ denotes complement of the set $A$.

4.2.1. Simulating $\left\{n_{k-1}<\tau_{b} \leq n_{k}, A_{k}\right\}$. We prescribe the following two step procedure: Let $q_{k}(b):=\sum_{i=n_{k-1}+1}^{n_{k}} \bar{F}(b+i \mu)$.

1. Choose an index $J \in\left\{n_{k-1}+1, \ldots, n_{k}\right\}$ such that $\mathbb{P}\{J=n\}=\bar{F}(b+$ $n \mu) / q_{k}(b)$, for $n_{k-1}<n \leq n_{k}$.

2. Simulate the increment $X_{n}$ from $F\left(\cdot \mid X_{n} \geq b+n \mu\right)$, if $n=J$; otherwise, simulate $X_{n}$ from $F(\cdot)$, for any $n \leq n_{k}$.

In this sampling procedure, we induce the 'big' jumps typically responsible for the occurrence of $\left\{n_{k-1}<\tau_{b} \leq n_{k}\right\}$ with suitable probabilities by sampling from the conditional distribution $F\left(\cdot \mid X_{J} \geq b+J \mu\right)$. This sampling 
procedure results in the importance sampling measure $\mathbb{P}_{k, 1}(\cdot)$ characterised by:

$$
d \mathbb{P}_{k, 1}\left(x_{1}, \ldots, x_{n_{k}}\right):=\sum_{i=n_{k-1}+1}^{n_{k}} \frac{\bar{F}(b+i \mu)}{q_{k}(b)} \cdot \frac{d F\left(x_{1}\right) \ldots d F\left(x_{n_{k}}\right)}{\bar{F}(b+i \mu)} \mathbf{1}\left(x_{i} \geq b+i a\right) .
$$

This in turn yields a likelihood ratio,

$$
\frac{d \mathbb{P}}{d \mathbb{P}_{k, 1}}\left(X_{1}, \ldots, X_{n_{k}}\right)=\frac{q_{k}(b)}{\#\left\{X_{i} \geq b+i \mu: n_{k-1}<i \leq n_{k}\right\}},
$$

on the set $A_{k}$. Then we have,

$$
Z_{k, 1}(b):=\frac{q_{k}(b)}{\#\left\{X_{i} \geq b+i \mu: n_{k-1}<i \leq n_{k}\right\}} \mathbb{I}\left(n_{k-1}<\tau_{b} \leq n_{k}, A_{k}\right)
$$

as the unbiased estimator for the quantity $\mathbb{P}\left\{n_{k-1}<\tau_{b} \leq n_{k}, A_{k}\right\}$. Here note that $\mathbb{I}\left(n_{k-1}<\tau_{b} \leq n_{k}, A_{k}\right)=1$ a.s. under $\mathbb{P}_{k, 1}(\cdot)$.

4.2.2. Simulating $\left\{n_{k-1}<\tau_{b} \leq n_{k}, B_{k}\right\}$. On the event $B_{k}$, none of the random variables $X_{1}, \ldots, X_{n_{k}}$ exceed the level $\left(b+n_{k-1} \mu\right)$; since these increments are bounded (on $B_{k}$ ), we can draw their samples from an appropriately truncated, exponentially twisted variation of $F(\cdot)$, as in Section 3.2, without losing absolute continuity on $\left\{n_{k-1}<\tau_{b} \leq n_{k}, B_{k}\right\}$. For estimating $\mathbb{P}\left\{n_{k-1}<\tau_{b} \leq n_{k}, B_{k}\right\}$, we draw samples of $X_{1}, \ldots, X_{\tau_{b} \wedge n_{k}}$ independently from the distribution $F_{k}(\cdot)$ satisfying,

$$
\begin{aligned}
\frac{d F_{k}(x)}{d F(x)} & =\exp \left(\theta_{k} x-\Lambda_{k}\left(\theta_{k}\right)\right) \mathbf{1}\left(x<b+n_{k-1} \mu\right), \quad x \in \mathbb{R} ; \\
\text { here, } \theta_{k}\left(=\theta_{k}(b)\right) & :=\frac{-\log \left(n_{k} \bar{F}\left(b+n_{k-1} \mu\right)\right)}{b+n_{k-1} \mu}, \text { and } \\
\Lambda_{k}(\theta) & :=\log \left(\int_{-\infty}^{b+n_{k-1} \mu} \exp \left(\theta_{k} x\right) F(d x)\right), \quad \theta \geq 0 .
\end{aligned}
$$

Let $\mathbb{P}_{k, 2}(\cdot)$ be the measure induced by drawing samples as above. Then the resulting likelihood ratio on $\left\{n_{k-1}<\tau_{b} \leq n_{k}, B_{k}\right\}$ is:

$$
\frac{d \mathbb{P}}{d \mathbb{P}_{k, 2}}\left(X_{1}, \ldots, X_{n_{k}}\right)=\exp \left(-\theta_{k} S_{\tau_{b}}+\tau_{b} \Lambda_{k}\left(\theta_{k}\right)\right) .
$$

The associated estimator for computing $\mathbb{P}\left\{n_{k-1}<\tau_{b} \leq n_{k}, B_{k}\right\}$ is:

$$
Z_{k, 2}(b):=\exp \left(-\theta_{k} S_{\tau_{b}}+\tau_{b} \Lambda_{k}\left(\theta_{k}\right)\right) \mathbb{I}\left(n_{k-1}<\tau_{b} \leq n_{k}, B_{k}\right)
$$


4.2.3. Simulating $\left\{n_{k-1}<\tau_{b} \leq n_{k}, \bar{A}_{k} \cap \bar{B}_{k}\right\}$. We draw samples in a two step procedure similar to that in the Section 4.2.1.

1. Choose an index $J$ uniformly at random from $\left\{1, \ldots, n_{k}\right\}$

2. Simulate the increment $X_{n}$ from $F\left(\cdot \mid X_{n} \geq b+n_{k-1} \mu\right)$, if $n=J$; otherwise, simulate $X_{n}$ from $F(\cdot)$, for any $n \leq n_{k}$.

If $\mathbb{P}_{k, 3}(\cdot)$ denotes the change of measure induced by drawing samples according to the above procedure, then the likelihood ratio on the set $\left\{n_{k-1}<\right.$ $\left.\tau_{b} \leq n_{k}, \bar{A}_{k} \cap \bar{B}_{k}\right\}$ is:

$$
\frac{d \mathbb{P}}{d \mathbb{P}_{k, 3}}\left(X_{1}, \ldots, X_{n_{k}}\right)=\frac{n_{k} \bar{F}\left(b+n_{k-1} \mu\right)}{\#\left\{X_{i} \geq b+n_{k-1} \mu: 1<i \leq n_{k}\right\}} .
$$

The resulting estimator for the computation of $\mathbb{P}\left\{n_{k-1}<\tau_{b} \leq n_{k}, \bar{A}_{k} \cap \bar{B}_{k}\right\}$ is:

$$
Z_{k, 3}(b):=\frac{n_{k} \bar{F}\left(b+n_{k-1} \mu\right)}{\#\left\{X_{i} \geq b+n_{k-1} \mu: 1<i \leq n_{k}\right\}} \mathbb{I}\left(n_{k-1}<\tau_{b} \leq n_{k}, \bar{A}_{k} \cap \bar{B}_{k}\right) .
$$

As in Section 3, the estimator for $\mathbb{P}\left\{n_{k-1}<\tau_{b} \leq n_{k}\right\}$ can be obtained by summing the estimators of component events $\mathbb{P}\left\{n_{k-1}<\tau_{b} \leq n_{k}, A_{k}\right\}, \mathbb{P}\left\{n_{k-1}<\right.$ $\left.\tau_{b} \leq n_{k}, B_{k}\right\}$, and $\mathbb{P}\left\{n_{k-1}<\tau_{b} \leq n_{k}, \bar{A}_{k} \cap \bar{B}_{k}\right\}$ :

$$
Z_{k}(b):=Z_{k, 1}(b)+Z_{k, 2}(b)+Z_{k, 3}(b) .
$$

4.3. Simulation of $\left\{\tau_{b}<\infty\right\}$ - the finite variance case. Here we develop on the ideas stated at the beginning of Section 4 . We have the increasing sequence of integers $\left(n_{k}: k \geq 0\right)$,

$$
n_{0}=0, n_{k}=r^{k} \text { for } k \geq 1,
$$

for some integer $r>1$. Further, we have an auxiliary random variable $K$ taking values in positive integers according to the probability mass function $\left(p_{k}: k \geq 1\right)$. As in (15), we re-express the quantity of interest as:

$$
\mathbb{P}\left\{\tau_{b}<\infty\right\}=\mathbb{E}\left[\mathbb{E}\left[\frac{\mathbb{P}\left\{n_{K-1}<\tau_{b} \leq n_{K}\right\}}{p_{K}} \mid K\right]\right] .
$$

From (25), we have estimators $\left\{Z_{k}(b): k \geq 1\right\}$ that can be used to compute the corresponding probabilities $\left\{\mathbb{P}\left\{n_{k-1}<\tau_{b} \leq n_{k}\right\}: k \geq 1\right\}$. Consider the following simulation procedure:

1. Draw a sample of $K$ such that $\mathbb{P}\{K=k\}=p_{k}$. 
2. Conditional on the realized value of $K$,

2a) Generate a realization of $Z_{K}(b)$ as in Section 4.2 .

2b) Return $Z_{K}(b) / p_{K}$.

We present the sample mean of the values returned by $N$ independent simulation runs of the above procedure as our final estimate of $\mathbb{P}\left\{\tau_{b}<\infty\right\}$. Let $Q(\cdot)$ denote the probability measure in the path space induced by the generation of increment random variables as a result of this sampling procedure; let $\mathbb{E}^{Q}[\cdot]$ and $\operatorname{Var}^{Q}[\cdot]$ be the expectation and variance operator associated with the measure $Q(\cdot)$. Given $b>0$, the overall unbiased estimator for the computation of $\mathbb{P}\left\{\tau_{b}<\infty\right\}$ is,

$$
Z(b):=\frac{Z_{K}(b)}{p_{K}}
$$

Note that the number of independent simulation runs needed to achieve a desired relative precision, as in (1), is directly related to the sampling variance of $Z(b)$. If $(Z(b): b>0)$ offer asymptotically vanishing relative error, we just need $o\left(\epsilon^{-2} \delta^{-1}\right)$ independent replications of the estimator. However, as pointed in [29], and further justified in [28], both the variance of an estimator and the expected computational effort required to generate a single sample are important performance measures, and their product can be considered as a 'figure of merit' in comparing performance of algorithms that provide unbiased estimators of $\mathbb{P}\left\{\tau_{b}<\infty\right\}$. For any given $b$, let $\nu_{b}$ denote the largest index of the increment random variables $\left(X_{n}: n \geq 1\right)$ considered for simulation in a particular simulation run. The expectation of $\nu_{b}$, then gives a measure of the expected number of increment random variables generated, and subsequently of the expected computational effort in every simulation run. In particular, the latter may be bounded from above by a constant $C>0$ times the expectation of $\nu_{b}$.

In a single run of the above procedure, if the realized value of $K$ is $k$, we look for estimating $\mathbb{P}\left\{n_{k-1}<\tau_{b} \leq n_{k}\right\}$ which does not entail the generation of more than $n_{k}$ increment random variables, thus ensuring termination. In particular, $n_{K-1} \leq \nu_{b} \leq n_{K}$. The following theorems give a measure of both the variance and the expected computational effort per replication of $Z(b)$ for a specific choice of the probabilities $p_{k}$. Recall that $Q(\cdot)$ is the probability measure that governs the law of $Z(b)$ when the random variables $Z_{K, j}(b)$ are generated as explained in Sections 4.2.1, 4.2.2 and 4.2.3.

In all the theorems that follow it is assumed that the common distribution $F(\cdot)$ of the increments satisfy Assumptions 1 and 2 . 
Theorem 2. For

$$
p_{k}=\frac{\bar{F}_{I}\left(b+n_{k-1} \mu\right)-\bar{F}_{I}\left(b+n_{k} \mu\right)}{\bar{F}_{I}(b)}, k \geq 1,
$$

the family of unbiased estimators $(Z(b): b>0)$ achieves asymptotically vanishing relative error for the computation of $\mathbb{P}\left\{\tau_{b}<\infty\right\}$, as $b \nearrow \infty$; that is:

$$
\varlimsup_{b \rightarrow \infty} \frac{\operatorname{Var}^{Q}[Z(b)]}{\mathbb{P}\left\{\tau_{b}<\infty\right\}^{2}}=0 .
$$

THEOREM 3 . If $\bar{F}(\cdot)$ is regularly varying with index $\alpha>2$, for the choice of $p=\left(p_{k}: k \geq 1\right)$ in $(26)$ :

$$
\mathbb{E}^{Q}\left[\nu_{b}\right] \leq \frac{r+o(1)}{\mu(\alpha-2)} b \text {, as } b \nearrow \infty .
$$

Proofs of both these results are given later in Section 5 .

REMARK 5. From Theorem 2, we have the vanishing relative error property for computing $\mathbb{P}\left\{\tau_{b}<\infty\right\}$ whenever the increment random variables $X_{n}$ have finite mean (irrespective of the variance). Therefore we require only $o\left(\epsilon^{-2} \delta^{-1}\right)$ i.i.d replications of $Z(b)$ to arrive at estimators that differ relatively at most by $\epsilon$ with probability at least $1-\delta$. Now from Theorem 3 we conclude that, if the tail index $\alpha>2$ (in which case the increments have finite variance), our importance sampling methodology estimates $\mathbb{P}\left\{\tau_{b}<\infty\right\}$ in $O(b)$ expected computational effort.

REMARK 6. From the conditional limit result in (19), one can infer that the values $p_{k}$ as in (26) roughly match the zero-variance probability $\mathbb{P}\left\{n_{k-1}<\tau_{b} \leq n_{k} \mid \tau_{b}<\infty\right\}$ asymptotically. For tails $\bar{F}(\cdot)$ with regularly varying index $1<\alpha<2$, we have that $\mathbb{E}\left[\tau_{b} \mid \tau_{b}<\infty\right]=\infty$; that is, the zero-variance measure itself has infinite expected termination time! Since $p_{k}$ are assigned a value similar to $\mathbb{P}\left\{n_{k-1}<\tau_{b} \leq n_{k} \mid \tau_{b}<\infty\right\}$, one might suspect infinite expected termination time for a single run of Algorithm 1 as well. As we note later in Remark 10 after proof of Theorem 3, for $p_{k} \mathrm{~s}$ as in (26), this is indeed the case. 
4.4. Simulation of $\left\{\tau_{b}<\infty\right\}$ - the infinite variance case. As indicated in Remark 6, infinite termination time for a simulation algorithm is clearly unacceptable. The following question then is natural: By choosing $p_{k}$ s differently, even if it means compromising on variance of the estimator, can one achieve finite expected termination time for the procedure in Section 4.3? Before answering this question below, we introduce a family of tail distributions and their integrated counterparts: for any $\beta>2$, define

$$
\bar{G}^{(\beta)}(x):=\frac{\bar{F}(x)}{x^{\beta-\alpha}} \text {, and } \bar{G}_{I}^{(\beta)}(x):=\int_{x}^{\infty} \bar{G}^{(\beta)}(u) d u \text {. }
$$

THEOREM 4. If the tail $\bar{F}(\cdot)$ is regularly varying with index $\alpha \in(1.5,2]$, then for any $\beta \in(2,2 \alpha-1)$,

$$
p_{k}=\frac{\bar{G}_{I}^{(\beta)}\left(b+n_{k-1} \mu\right)-\bar{G}_{I}^{(\beta)}\left(b+n_{k} \mu\right)}{\bar{G}_{I}^{(\beta)}(b)}, k \geq 1
$$

yields a family of unbiased estimators $\left(Z(b)=Z_{K}(b) / p_{K}: b>0\right)$ achieving

1. strong efficiency: $\varlimsup_{b \rightarrow \infty} \frac{\operatorname{Var}^{Q}[Z(b)]}{\mathbb{P}\left\{\tau_{b}<\infty\right\}^{2}}<\infty$, and

2. finite expected termination time: $\mathbb{E}^{Q}\left[\nu_{b}\right] \leq \frac{r+o(1)}{\mu(\beta-2)} b$, as $b \nearrow \infty$.

REMARK 7. Because of the strong efficiency, we need just $O\left(\epsilon^{-2} \delta^{-1}\right)$ i.i.d. replications of $Z(b)$ to achieve the desired relative precision. As in Remark 5 , due to the bound on $\mathbb{E}\left[\nu_{b}\right]$ in Theorem 4 , the average computational effort for the entire estimation procedure is just $O\left(\epsilon^{-2} \delta^{-1} b\right)$. It is important to see this achievement in the context of Remark 6 : the induced measure $Q(\cdot)$ deviates from the zero-variance measure such that we get finite expected termination time, but only at the cost of losing vanishing relative error property to strong efficiency. Thus for the selection of $p_{k} \mathrm{~s}$ as in (28), the suggested procedure ends up offering superior performance (in terms of computational complexity) compared to the algorithms that tend to just approximate the zero-variance measure.

Given this result, it is difficult not to wonder why the tail index $\alpha$ should be larger than 1.5 in the statement of Theorem 4 , and what happens when $\alpha \leq 1.5$. The following result shows that it is indeed impossible to have both strong efficiency and finite expected termination time when the tail index $\alpha<1.5$. 
THEOREM 5. If the tail index $\alpha<1.5$, there does not exist an assignment of $\left(p_{k}, n_{k}: k \geq 1\right)$ such that both $\mathbb{E}^{Q}\left[Z^{2}(b)\right]$ and $\mathbb{E}^{Q}\left[\nu_{b}\right]$ are simultaneously finite.

REMARK 8. If the tail index $\alpha=1.5$, the possibility of having both $\mathbb{E}^{Q}\left[Z^{2}(b)\right]$ and $\mathbb{E}^{Q}\left[\nu_{b}\right]$ finite will depend on the slowly varying function $L(\cdot)$. As we shall see in the proof of Theorem 5 ,

$$
\left.\mathbb{E}^{Q}\left[Z^{2}(b)\right] \mathbb{E}^{Q}\left[\nu_{b}\right]=\Omega\left(\int_{b^{2}}^{\infty} \sqrt{u} \bar{F}(u) d u\right)\right)
$$

as $b \nearrow \infty$. If $L(x)=O\left((\log x)^{-m}\right), m \geq 2$, the above integral is finite, whereas if $L(x)=O(\log x)$ it is infinite; and it easily verified that the case of $L(x)=O\left((\log x)^{-m}\right), m \geq 2$, goes through the proof of Theorem 4, thus achieving both strong efficiency and finite expected termination time. This illustrates the subtle dependence on the associated slowly varying function $L(\cdot)$ for the existence of such $p_{k} \mathrm{~s}$ and $n_{k} \mathrm{~s}$.

As illustrated by the theorem below, for $\alpha \in(1,1.5]$, we still have algorithms that demand only $O(b)$ units of expected computer time if we look for less stringent notions of efficiency.

THeOREM 6. If the tail $\bar{F}(\cdot)$ is regularly varying with index $\alpha \in(1,1.5]$, then there exists an explicit selection of $p=\left(p_{k}: k \geq 1\right)$ such that the family of unbiased estimators $(Z(b): b>0)$ satisfies both:

$$
\begin{gathered}
\varlimsup_{b \rightarrow \infty} \frac{\mathbb{E}^{Q}\left[Z^{1+\gamma}(b)\right]}{\mathbb{P}\left\{\tau_{b}<\infty\right\}^{1+\gamma}}<\infty \text { for all } \gamma \in\left(0, \frac{\alpha-1}{2-\alpha}\right), \text { and } \\
\mathbb{E}^{Q}\left[\nu_{b}\right] \leq C b \text { for some constant } C .
\end{gathered}
$$

In particular, for the following selection of $p=\left(p_{k}: k \geq 1\right)$,

$$
p_{k}=\frac{\bar{G}_{I}^{(\beta)}\left(b+n_{k-1} \mu\right)-\bar{G}_{I}^{(\beta)}\left(b+n_{k} \mu\right)}{\bar{G}_{I}^{(\beta)}(b)}, k \geq 1
$$

if $\beta$ is chosen in $\left(2, \alpha+\gamma^{-1}(\alpha-1)\right)$, both the above inequalities are satisfied.

REMARK 9. If the estimator $Z(b)$ satisfies (29), similar to how we arrived at (1), it can be shown that $O\left(\epsilon^{-\left(1+\gamma^{-1}\right)} \delta^{-\gamma^{-1}}\right)$ i.i.d. replications of $Z(b)$ are enough to produce estimates having relative error at most $\epsilon$ with probability at least $1-\delta$. Now according to Theorem 6 , the expected termination time 
in each replication is $O(b)$. Thus with the $p_{k}$ s chosen as in (30), we expend just $O\left(\epsilon^{-\left(1+\gamma^{-1}\right)} \delta^{-\gamma^{-1}} b\right)$ units of computer time on an average, which is still linear in $b$. The price we pay by not adhering to strong efficiency is the worse dependence on the parameters $\epsilon$ and $\delta$.

It is further interesting to note that a vastly different state-dependent methodology developed using Lyapunov inequalities in [14] also hits identical barriers and provides results similar to ours: They present algorithms that are both strongly efficient and possess $O(b)$ expected termination time for the case of tails having index $\alpha>1.5$; whereas when $\alpha \in(1,1.5]$, they provide estimators satisfying $(29)$ along with $O(b)$ expected termination time of a simulation run.

5. Proofs of key theorems. For proving Theorems 2, 4 and 6 , which are on the efficiency of estimators $\{Z(b): b>0\}$, we first present a result pertaining to the efficiency of component estimators $\left\{Z_{k}(b): k \geq 1\right\}$. Recall from Section 4.2 that

$$
Z_{k}(b):=Z_{k, 1}(b)+Z_{k, 2}(b)+Z_{k, 3}(b)
$$

is an unbiased estimator for $\mathbb{P}\left\{n_{k-1}<\tau_{b} \leq n_{k}\right\}$, and

$$
q_{k}(b):=\sum_{j=n_{k-1}+1}^{n_{k}} \bar{F}(b+j \mu) .
$$

To aid the analysis of second moment of estimators $Z_{k}(b)$, let $\mathbb{P}_{k}(\cdot)$ denote the composite measure induced due to the simulation of random variables $Z_{k, j}, j=1,2,3$ independently according to measures $\mathbb{P}_{k, j}, j=1,2,3$, respectively. Let $\mathbb{E}_{k}[\cdot]$ denote the corresponding expectation operator.

THEOREM 7. Under Assumptions 1 and 2, the family of estimators $\left\{Z_{k}(b): k \geq 1, b>0\right\}$ satisfies the following as $b \nearrow \infty$ :

$$
\sup _{k: n_{k}<b^{\eta}} \frac{\mathbb{E}_{k}\left[Z_{k}^{2}(b)\right]}{q_{k}^{2}(b)} \leq 1+o(1) \text { and } \sup _{k: n_{k} \geq b^{\eta}} \frac{\mathbb{E}_{k}\left[Z_{k}^{2}(b)\right]}{q_{k}^{2}(b)} \leq c
$$

for some $c>0$ and $\eta>1$.

We prove Theorem 7 by analysing the second moment of estimators $Z_{k, 1}(\cdot), Z_{k, 2}(\cdot)$ and $Z_{k, 3}(\cdot)$ separately in the Lemmas 2,6 and 7 below. 
Lemma 2. Under Assumption 1,

$$
\sup _{k} \frac{\mathbb{E}_{k, 1}\left[Z_{k, 1}^{2}(b)\right]}{q_{k}^{2}(b)} \leq 1 .
$$

Proof. Recall that $\mathbb{P}_{k, 1}(\cdot)$ is the measure resulting due to the simulation of increments as in the two-step procedure specified in Section 4.2.1. Since the quantity $\#\left\{X_{i} \geq b+i \mu: n_{k-1}<i \leq n_{k}\right\}$ is at least 1 when the increments are generated from $\mathbb{P}_{k, 1}(\cdot)$, we have $Z_{k, 1}(b) \leq q_{k}(b)$. Therefore,

$$
\mathbb{E}_{k, 1}\left[Z_{k, 1}^{2}(b)\right] \leq q_{k}^{2}(b),
$$

which proves the claim.

For a similar analysis on the second moment of estimators $Z_{k, 2}(b)$ and $Z_{k, 3}(b)$, we need the following results which are proved in the appendix.

LEMma 3. Under Assumption 1, there exists a constant $c_{1}>1$ such that $\exp \left(n_{k} \Lambda_{k}\left(\theta_{k}\right)\right) \leq c_{1}$ for all $k, b$.

Lemma 4. Under Assumption 1, there exists a positive constant $c_{2}$ such that,

$$
\sup _{k \geq 1, b>0} \frac{n_{k} \bar{F}\left(b+n_{k-1} \mu\right)}{q_{k}(b)} \leq c_{2} .
$$

Proposition 2. Under Assumption 1,

$$
\sup _{k \geq 1}\left|\frac{\mathbb{P}\left\{n_{k-1}<\tau_{b} \leq n_{k}, A_{k}\right\}}{q_{k}(b)}-1\right|=O\left(b^{-\frac{\alpha-1}{2 \alpha}}\right),
$$

as $b \nearrow \infty$.

Lemma 5. Under Assumptions 1 and 2, there exist constants $\eta>1$ and $c_{3}$ such that,

$$
\begin{aligned}
& \sup _{k: n_{k}<b^{\eta}} \frac{\mathbb{P}\left\{n_{k-1}<\tau_{b} \leq n_{k}, \bar{A}_{k}\right\}}{q_{k}(b)}=o(1) \text { and } \\
& \sup _{k: n_{k} \geq b^{\eta}} \frac{\mathbb{P}\left\{n_{k-1}<\tau_{b} \leq n_{k}, \bar{A}_{k}\right\}}{q_{k}(b)} \leq c_{3},
\end{aligned}
$$

as $b \nearrow \infty$. 
Using Lemmas 3, 4 and 5, we now present an asymptotic analysis on the second moment of estimators $Z_{k, 2}(\cdot)$ and $Z_{k, 3}(\cdot)$.

Lemma 6. Under Assumptions 1 and 2, as $b \nearrow \infty$,

$$
\sup _{k: n_{k}<b^{\eta}} \frac{\mathbb{E}_{k, 2}\left[Z_{k, 2}^{2}(b)\right]}{q_{k}^{2}(b)}=o(1) \text { and } \sup _{k: n_{k} \geq b^{\eta}} \frac{\mathbb{E}_{k, 2}\left[Z_{k, 2}^{2}(b)\right]}{q_{k}^{2}(b)} \leq c_{4}
$$

for some positive constant $c_{4}$.

Proof. Since $\tau_{b} \leq n_{k}$ on the event $\left\{n_{k-1}<\tau_{b} \leq n_{k}\right\}$,

$$
\exp \left(\tau_{b} \Lambda_{k}\left(\theta_{k}\right)\right) \mathbb{I}\left(n_{k-1}<\tau_{b} \leq n_{k}, B_{k}\right) \leq c_{1},
$$

because of Lemma 3. Further note that $\theta_{k} S_{\tau_{b}} \geq-\log \left(n_{k} \bar{F}\left(b+n_{k-1} \mu\right)\right)$ on $\left\{n_{k-1}<\tau_{b} \leq n_{k}\right\}$. Therefore from (23),

$$
Z_{k, 2}(b) \leq c_{1}\left(n_{k} \bar{F}\left(b+n_{k-1} \mu\right)\right) \mathbb{I}\left(n_{k-1}<\tau_{b} \leq n_{k}, B_{k}\right) \text {, for all } k .
$$

Now changing the expectation operator in the evaluation of second moment of the estimator results in the following bound: for all $k$,

$$
\begin{gathered}
\mathbb{E}_{k, 2}\left[Z_{k, 2}^{2}(b)\right]=\mathbb{E}\left[Z_{k, 2}(b)\right] \leq c_{1}\left(n_{k} \bar{F}\left(b+n_{k-1} \mu\right)\right) \mathbb{P}\left\{n_{k-1}<\tau_{b} \leq n_{k}, B_{k}\right\} . \\
\text { Therefore } \frac{\mathbb{E}_{k, 2}\left[Z_{k, 2}^{2}(b)\right]}{q_{k}^{2}(b)} \leq c_{1} \frac{\left(n_{k} \bar{F}\left(b+n_{k-1} \mu\right)\right)}{q_{k}(b)} \frac{\mathbb{P}\left\{n_{k-1}<\tau_{b} \leq n_{k}, \bar{A}_{k}\right\}}{q_{k}(b)} .
\end{gathered}
$$

Then it follows from Lemmas 4 and 5 that, as $b \nearrow \infty$,

$$
\sup _{k: n_{k}<b^{\eta}} \frac{\mathbb{E}_{k, 2}\left[Z_{k, 2}^{2}(b)\right]}{q_{k}^{2}(b)}=o(1), \text { and } \sup _{k: n_{k} \geq b^{\eta}} \frac{\mathbb{E}_{k, 2}\left[Z_{k, 2}^{2}(b)\right]}{q_{k}^{2}(b)} \leq c_{1} c_{2} c_{3}=: c_{4}<\infty,
$$

thus proving the claim.

Lemma 7. Under Assumptions 1 and 2, as $b \nearrow \infty$,

$$
\sup _{k: n_{k}<b^{\eta}} \frac{\mathbb{E}_{k, 3}\left[Z_{k, 3}^{2}(b)\right]}{q_{k}^{2}(b)}=o(1) \text { and } \sup _{k: n_{k} \geq b^{\eta}} \frac{\mathbb{E}_{k, 3}\left[Z_{k, 3}^{2}(b)\right]}{q_{k}^{2}(b)} \leq c_{5}
$$

for some positive constant $c_{5}$. 
Proof. When the increments are generated as prescribed in the two-step procedure in Section 4.2.3, we have $\#\left\{X_{i} \geq b+n_{k-1} \mu: 1<i \leq n_{k}\right\} \geq 1$, and hence,

$$
Z_{k, 3}(b) \leq n_{k} \bar{F}\left(b+n_{k-1} \mu\right) \mathbb{I}\left(n_{k-1}<\tau_{b} \leq n_{k}, \bar{A}_{k} \cap \bar{B}_{k}\right) .
$$

Now a bound on the second moment of the estimator can be obtained as before:

$\mathbb{E}_{k .3}\left[Z_{k, 3}^{2}(b)\right]=\mathbb{E}\left[Z_{k .3}(b)\right] \leq n_{k} \bar{F}\left(b+n_{k-1} \mu\right) \mathbb{P}\left\{n_{k-1}<\tau_{b} \leq n_{k}, \bar{A}_{k} \cap \bar{B}_{k}\right\}$.

Therefore $\frac{\mathbb{E}_{k, 3}\left[Z_{k, 3}^{2}(b)\right]}{q_{k}^{2}(b)} \leq \frac{\left(n_{k} \bar{F}\left(b+n_{k-1} \mu\right)\right)}{q_{k}(b)} \frac{\mathbb{P}\left\{n_{k-1}<\tau_{b} \leq n_{k}, \bar{A}_{k}\right\}}{q_{k}(b)}$.

Then it follows from Lemmas 4 and 5 that, as $b \nearrow \infty$,

$$
\sup _{k: n_{k}<b^{\eta}} \frac{\mathbb{E}_{k, 3}\left[Z_{k, 3}^{2}(b)\right]}{q_{k}^{2}(b)}=o(1), \text { and } \sup _{k: n_{k} \geq b^{\eta}} \frac{\mathbb{E}_{k, 3}\left[Z_{k, 3}^{2}(b)\right]}{q_{k}^{2}(b)} \leq c_{2} c_{3}=: c_{5}<\infty,
$$

thus establishing the claim.

Proof of Theorem $\%$. Since $\left\{Z_{k, i}(b): i=1,2,3\right\}$ are independent, for $i \neq 1$,

$$
\begin{aligned}
\frac{\mathbb{E}_{k}\left[Z_{k, 1}(b) Z_{k, i}(b)\right]}{q_{k}^{2}(b)} & =\frac{\mathbb{E}_{k, 1}\left[Z_{k, 1}(b)\right]}{q_{k}(b)} \frac{\mathbb{E}_{k, i}\left[Z_{k, i}(b)\right]}{q_{k}(b)} \\
& \leq \frac{\mathbb{P}\left\{n_{k-1}<\tau_{b} \leq n_{k}, A_{k}\right\}}{q_{k}(b)} \frac{\mathbb{P}\left\{n_{k-1}<\tau_{b} \leq n_{k}, \bar{A}_{k}\right\}}{q_{k}(b)} .
\end{aligned}
$$

Then from Proposition 2 and Lemma 5, we have that as $b \nearrow \infty$,

$$
\sup _{k: n_{k}<b^{\eta}} \frac{\mathbb{E}_{k}\left[Z_{k, 1}(b) Z_{k, i}(b)\right]}{q_{k}^{2}(b)}=o(1), \text { and } \sup _{k: n_{k} \geq b^{\eta}} \frac{\mathbb{E}_{k}\left[Z_{k, 1}(b) Z_{k, i}(b)\right]}{q_{k}^{2}(b)}<\infty \text {. }
$$

Similarly from Lemma 5 , as $b \nearrow \infty$,

$$
\sup _{k} \frac{\mathbb{E}_{k}\left[Z_{k, 2}(b) Z_{k, 3}(b)\right]}{q_{k}^{2}(b)} \leq \sup _{k} \frac{\mathbb{P}\left\{n_{k-1}<\tau_{b} \leq n_{k}, \bar{A}_{k}\right\}^{2}}{q_{k}^{2}(b)}=o(1) .
$$

Since $Z_{k}(b)=Z_{k, 1}(b)+Z_{k, 2}(b)+Z_{k, 3}(b)$, we have

$$
\mathbb{E}_{k}\left[Z_{k}^{2}(b)\right]=\sum_{i, j=1}^{3} \mathbb{E}_{k}\left[Z_{k, i}(b) Z_{k, j}(b)\right]
$$


Combining above observations with the results of Lemmas 2, 6 and 7, we conclude that as $b \nearrow \infty$,

$$
\sup _{k: n_{k}<b^{\eta}} \frac{\mathbb{E}_{k}\left[Z_{k}^{2}(b)\right]}{q_{k}^{2}(b)} \leq 1+o(1) \text { and } \sup _{k: n_{k} \geq b^{\eta}} \frac{\mathbb{E}_{k}\left[Z_{k}^{2}(b)\right]}{q_{k}^{2}(b)} \leq c
$$

for some positive constant $c$.

The following uniform bounds will be useful:

LemMa 8. For all $k \geq 1$,

$$
q_{k}(b) \leq \frac{1}{\mu}\left(\bar{F}_{I}\left(b+n_{k-1} \mu\right)-\bar{F}_{I}\left(b+n_{k} \mu\right)\right) .
$$

Further as $b \nearrow \infty$,

$$
q_{k}(b) \geq(1-o(1)) \frac{1}{\mu}\left(\bar{F}_{I}\left(b+n_{k-1} \mu\right)-\bar{F}_{I}\left(b+n_{k} \mu\right)\right),
$$

uniformly in $k$.

Proof. For any $k \geq 1$,

$q_{k}(b)=\sum_{i=n_{k-1}+1}^{n_{k}} \bar{F}(b+i \mu) \leq \sum_{i=n_{k-1}+1}^{n_{k}} \int_{i-1}^{i} \bar{F}(b+u \mu) d u=\int_{n_{k-1}}^{n_{k}} \bar{F}(b+u \mu) d u$.

Changing variables from $u$ to $v=b+u \mu$ results in,

$$
q_{k}(b) \leq \frac{1}{\mu} \int_{b+n_{k-1} \mu}^{b+n_{k} \mu} \bar{F}(v) d v
$$

which establishes the upper bound because $\bar{F}_{I}(x):=\int_{x}^{\infty} \bar{F}(u) d u$.

For the lower bound, see that

$$
\begin{aligned}
q_{k}(b) & =\sum_{i=n_{k-1}+1}^{n_{k}} \bar{F}(b+i \mu) \geq \sum_{i=n_{k-1}+1}^{n_{k}} \int_{i}^{i+1} \bar{F}(b+u \mu) d u \\
& =\int_{n_{k-1}+1}^{n_{k}+1} \bar{F}(b+u \mu) d u .
\end{aligned}
$$

Now after changing variables from $u$ to $v=b+u \mu$, we use the long-tailedness of $\bar{F}_{I}(\cdot)$ to see that, given $\epsilon>0$, for large values of $b$,

$$
\begin{aligned}
q_{k}(b) & \geq \frac{1}{\mu}\left(\bar{F}_{I}\left(b+\left(n_{k-1}+1\right) \mu\right)-\bar{F}_{I}\left(b+\left(n_{k}+1\right) \mu\right)\right) \\
& \geq(1-\epsilon) \frac{1}{\mu}\left(\bar{F}_{I}\left(b+n_{k-1} \mu\right)-\bar{F}_{I}\left(b+n_{k} \mu\right)\right)
\end{aligned}
$$

for all $k$. 
Proof of Theorem 2. Recall that the overall estimator is,

$$
Z(b)=\frac{Z_{K}(b)}{p_{K}}
$$

where $p_{k}$ is as in (26). Second moment of the estimator $Z(b)$ is bounded as below:

$$
\begin{aligned}
\mathbb{E}^{Q}\left[Z^{2}(b)\right]=\mathbb{E}^{Q} & {\left[\left(\frac{Z_{K}(b)}{p_{K}}\right)^{2}\right] } \\
=\mathbb{E}^{Q} & {\left[\mathbb{E}^{Q}\left[\frac{Z_{K}^{2}(b)}{q_{K}^{2}(b)} \frac{q_{K}^{2}(b)}{p_{K}^{2}} ; n_{K}<b^{\eta} \mid K\right]\right] } \\
& +\mathbb{E}^{Q}\left[\mathbb{E}^{Q}\left[\frac{Z_{K}^{2}(b)}{q_{K}^{2}(b)} \frac{q_{K}^{2}(b)}{p_{K}^{2}} ; n_{K} \geq b^{\eta} \mid K\right]\right]
\end{aligned}
$$

From the definition of $p_{k}$ and Lemma 8 , we have $q_{K}^{2}(b) \leq \bar{F}_{I}^{2}(b) p_{K}^{2}$. Combining this with Theorem 7 it follows that,

$$
\begin{aligned}
\frac{\mathbb{E}^{Q}\left[Z^{2}(b)\right]}{\bar{F}_{I}^{2}(b)} & \leq \mathbb{E}^{Q}\left[\mathbb{E}^{Q}\left[\frac{Z_{K}^{2}(b)}{q_{K}^{2}(b)} ; n_{K}<b^{\eta} \mid K\right]\right]+\mathbb{E}^{Q}\left[\mathbb{E}^{Q}\left[\frac{Z_{K}^{2}(b)}{q_{K}^{2}(b)} ; n_{K} \geq b^{\eta} \mid K\right]\right] \\
& \leq 1+o(1)+c \mathbb{P}\left\{n_{K} \geq b^{\eta}\right\} \\
& \leq 1+o(1)+O\left(\frac{\bar{F}_{I}\left(b+b^{\eta}\right)}{\bar{F}_{I}(b)}\right)=1+o(1),
\end{aligned}
$$

as $b \nearrow \infty$. The last inequality follows from observing that $\mathbb{P}\left\{n_{K} \geq b^{\eta}\right\}=$ $\sum_{k: n_{k} \geq b^{\eta}} p_{k}$. Since $\eta>1$, we have the asymptotically vanishing relative error property of the estimators $(Z(b): b>0)$.

Proof of Theorem 3. Recall that $\nu_{b}$ denotes the maximum of indices of the increment random variables $\left(X_{i} \mathrm{~s}\right)$ considered for simulation in a particular simulation run. From the sampling procedures in Section 4.2, it is clear that $\nu_{b} \leq n_{K}$. Therefore,

$$
\begin{aligned}
\mathbb{E}^{Q}\left[\nu_{b}\right] & \leq \sum_{k \geq 1} p_{k} n_{k} \\
& =r p_{1}+\sum_{k \geq 2} r^{k} p_{k} \\
& =\frac{1}{\bar{F}_{I}(b)}\left(r \int_{b}^{b+r \mu} \bar{F}(u) d u+\sum_{k \geq 1} r^{k+1} \int_{b+r^{k} \mu}^{b+r^{k+1} \mu} \bar{F}(u) d u\right) .
\end{aligned}
$$




$$
\begin{aligned}
\text { Since } r^{k} \int_{b+r^{k} \mu}^{b+r^{k+1} \mu} & \bar{F}(u) d u=\frac{b+r^{k} \mu-b}{\mu} \int_{b+r^{k} \mu}^{b+r^{k+1} \mu} \bar{F}(u) d u \\
& \leq \frac{1}{\mu}\left(\int_{b+r^{k} \mu}^{b+r^{k+1} \mu} u \bar{F}(u) d u-b \int_{b+r^{k} \mu}^{b+r^{k+1} \mu} \bar{F}(u) d u\right)
\end{aligned}
$$

we write $\sum_{k \geq 1} r^{k+1} \int_{b+r^{k} \mu}^{b+r^{k+1} \mu} \bar{F}(u) d u$

$$
\begin{aligned}
& \leq \frac{r}{\mu} \sum_{k \geq 1}\left(\int_{b+r^{k} \mu}^{b+r^{k+1} \mu} u \bar{F}(u) d u-b \int_{b+r^{k} \mu}^{b+r^{k+1} \mu} \bar{F}(u) d u\right) \\
& =\frac{r}{\mu}\left(\int_{b+r \mu}^{\infty} u \bar{F}(u) d u-\int_{b+r \mu}^{\infty} \bar{F}(u) d u\right) \\
& \leq \frac{r+o(1)}{\mu}\left(\frac{(b+r \mu)^{2}}{\alpha-2}-b \frac{b+r \mu}{\alpha-1}\right) \bar{F}(b+r \mu), \\
& =\frac{r+o(1)}{\mu(\alpha-1)(\alpha-2)} b^{2} \bar{F}(b), \text { as } b \nearrow \infty .
\end{aligned}
$$

where the penultimate step follows from Karamata's theorem (see (5)), and the final step just uses long-tailed nature of $\bar{F}(\cdot)$. Also note that: $\int_{b}^{b+r \mu} \bar{F}(u) d u \leq r \mu \bar{F}(b)$, and by application of Karamata's theorem, we have $\bar{F}_{I}(b) \sim b F(b) /(\alpha-1)$, as $b \nearrow \infty$. Therefore from (33),

$$
\mathbb{E}^{Q}\left[\nu_{b}\right] \leq \frac{r+o(1)}{\mu(\alpha-2)} b, \text { as } b \nearrow \infty
$$

thus yielding the required bound on the expected termination time.

REMARK 10. Similar to how we arrived at (34), lower bounds can be obtained to show that $\mathbb{E}^{Q}\left[\nu_{b}\right]=\Omega\left(\int_{b}^{\infty} u \bar{F}(u) d u\right)$. If the tail index $\alpha<$ $2, \int_{b}^{\infty} u \bar{F}(u) d u$ turns out to be infinite, and subsequently $\mathbb{E}^{Q}\left[\nu_{b}\right]=\infty$. Though the assignment of $p_{k} \mathrm{~s}$ in (26) yields vanishing relative error for any $\alpha>1$, it fails to provide algorithms which have finite expected termination time when the increment random variables $X$ have infinite variance (e.g., when $\alpha<2$ ), thus making this choice of $p_{k}$ not suitable for practice.

Proof of Theorem 4. We obtain upper bounds for both the variance of the estimator $Z(b)$ and the expected termination time. 
1. Variance of $Z(b)$ : Since $Q(K=k)=p_{k}$,

$$
\begin{aligned}
\mathbb{E}^{Q}\left[Z^{2}(b)\right] & =\mathbb{E}^{Q}\left[\frac{Z_{K}^{2}(b)}{p_{K}^{2}}\right]=\sum_{k} p_{k} \frac{\mathbb{E}^{Q}\left[Z_{k}^{2}(b)\right]}{p_{k}^{2}} \\
& =\sum_{k} \frac{\mathbb{E}^{Q}\left[Z_{k}^{2}(b)\right]}{q_{k}^{2}(b)} \frac{q_{k}^{2}(b)}{p_{k}} .
\end{aligned}
$$

Following Lemma 8 and the assignment of $p_{k}$ s as in (28), we can write,

$$
\frac{q_{k}(b)}{p_{k}} \leq \frac{\bar{F}_{I}\left(b+n_{k-1} \mu\right)-\bar{F}_{I}\left(b+n_{k} \mu\right)}{\bar{G}_{I}^{(\beta)}\left(b+n_{k-1} \mu\right)-\bar{G}_{I}^{(\beta)}\left(b+n_{k} \mu\right)} \bar{G}_{I}^{(\beta)}(b) .
$$

To obtain an upper bound, we note the following:

$$
\begin{aligned}
\bar{F}_{I}\left(b+n_{k-1} \mu\right)-\bar{F}_{I}\left(b+n_{k} \mu\right) & =\int_{b+n_{k-1} \mu}^{b+n_{k} \mu} \bar{F}(u) d u \\
& \leq\left(n_{k}-n_{k-1}\right) \mu \bar{F}\left(b+n_{k-1} \mu\right), \\
\bar{G}_{I}^{(\beta)}\left(b+n_{k-1} \mu\right)-\bar{G}_{I}^{(\beta)}\left(b+n_{k} \mu\right) & =\int_{b+n_{k-1} \mu}^{b+n_{k} \mu} \bar{G}^{(\beta)}(u) d u \\
& \geq\left(n_{k}-n_{k-1}\right) \mu \bar{G}^{(\beta)}\left(b+n_{k} \mu\right), \text { and } \\
\frac{\bar{G}^{(\beta)}\left(b+n_{k-1} \mu\right)}{\bar{G}^{(\beta)}\left(b+n_{k} \mu\right)} & \leq r^{\beta}+o(1), \text { as } b \nearrow \infty .
\end{aligned}
$$

The last inequality follows by observing that $b+n_{k} \mu \leq r\left(b+n_{k-1} \mu\right)$ and subsequently from the regularly varying nature of $\bar{G}^{(\beta)}(\cdot)$. Therefore as $b \nearrow$ $\infty$

$$
\begin{aligned}
\frac{q_{k}(b)}{p_{k}} & \leq \frac{\bar{G}^{(\beta)}\left(b+n_{k-1} \mu\right)}{\bar{G}^{(\beta)}\left(b+n_{k} \mu\right)} \frac{\bar{F}\left(b+n_{k-1} \mu\right)}{\bar{G}^{(\beta)}\left(b+n_{k-1} \mu\right)} \bar{G}_{I}^{(\beta)}(b) \\
& =\left(r^{\beta}+o(1)\right)\left(b+n_{k-1} \mu\right)^{\beta-\alpha} \bar{G}_{I}^{(\beta)}(b),
\end{aligned}
$$


for all $k$, because $\bar{F}(x) / \bar{G}^{(\beta)}(x)=x^{\beta-\alpha}$. Combining this with Theorem 7 , it follows from (36) that

$$
\begin{aligned}
\mathbb{E}^{Q}\left[Z^{2}(b)\right] & \leq\left(c r^{\beta}+o(1)\right) \bar{G}_{I}^{(\beta)}(b) \sum_{k}\left(b+n_{k-1} \mu\right)^{\beta-\alpha} q_{k}(b) \\
& \leq\left(c r^{\beta}+o(1)\right) \bar{G}_{I}^{(\beta)}(b) \sum_{k}\left(b+n_{k-1} \mu\right)^{\beta-\alpha} \int_{b+n_{k-1} \mu}^{b+n_{k} \mu} \bar{F}(u) d u \\
& \leq\left(c r^{\beta}+o(1)\right) \bar{G}_{I}^{(\beta)}(b) \sum_{k} \int_{b+n_{k-1} \mu}^{b+n_{k} \mu} u^{\beta-\alpha} \bar{F}(u) d u \\
& \leq\left(c r^{\beta}+o(1)\right) \bar{G}_{I}^{(\beta)}(b) \int_{b}^{\infty} u^{\beta-\alpha} \bar{F}(u) d u
\end{aligned}
$$

as $b \nearrow \infty$. Since $2 \alpha-\beta>1$, it follows from Karamata's theorem (cf. (5)) that

$$
\mathbb{E}^{Q}\left[Z^{2}(b)\right] \leq\left(c r^{\beta}+o(1)\right) \bar{G}_{I}^{(\beta)}(b) b^{\beta-\alpha+1} \frac{\bar{F}(b)}{2 \alpha-\beta-1}, \text { as } b \nearrow \infty .
$$

Further $(\alpha-1) \bar{F}_{I}(b) \sim b \bar{F}(b)$ and $b^{\beta-\alpha} \bar{G}_{I}^{(\beta)}(b) \sim \bar{F}_{I}(b)$, as $b \nearrow \infty$. Therefore,

$$
\varlimsup_{b \rightarrow \infty} \frac{\mathbb{E}^{Q}\left[Z^{2}(b)\right]}{\bar{F}_{I}^{2}(b)} \leq \frac{(\alpha-1) c r^{\beta}+o(1)}{2 \alpha-\beta-1}<\infty .
$$

Now since $\mathbb{P}\left\{\tau_{b}<\infty\right\} \sim \mu^{-1} \bar{F}_{I}(b)$, we have strong efficiency.

2. Expected termination time: Since $\nu_{b} \leq n_{K}, \mathbb{E}^{Q}\left[\nu_{b}\right] \leq \mathbb{E}^{Q}\left[n_{K}\right]=\sum_{k} p_{k} n_{k}$. For the choice of $p_{k}$ in (28), following exactly the same steps in the proof of Theorem 3, we arrive at:

$$
\mathbb{E}^{Q}\left[\nu_{b}\right] \leq \frac{r}{\mu}\left(\mu \int_{b}^{b+r \mu} \bar{G}^{(\beta)}(u) d u+\int_{b+r \mu}^{\infty} u \bar{G}^{(\beta)}(u) d u-b \int_{b+r \mu}^{\infty} \bar{G}^{(\beta)}(u) d u\right) .
$$

Since $\bar{G}^{(\beta)}(\cdot)$ is regularly varying with tail index larger than 2 , by application of Karamata's theorem, we have:

$$
\int_{b+r \mu}^{\infty} u \bar{G}^{(\beta)}(u) d u \sim \frac{(b+r \mu)^{2}}{\beta-2} \bar{G}^{(\beta)}(b+r \mu),
$$

which would not have been the case if we had persisted with using $\bar{F}_{I}(\cdot)$ instead of $\bar{G}_{I}^{(\beta)}(\cdot)$ for $p_{k}$. Again following the remaining steps in the proof of Theorem 3, we conclude that:

$$
\mathbb{E}^{Q}\left[\nu_{b}\right] \leq \frac{r+o(1)}{\mu(\beta-2)} b, \text { as } b \nearrow \infty,
$$


thus yielding finite termination time even when the zero-variance measure fails to offer this desirable property.

Proof of Theorem 5. Since $Q(K=k)=p_{k}$, see that:

$$
\mathbb{E}^{Q}\left[Z^{2}(b)\right]=\mathbb{E}^{Q}\left[\frac{Z_{K}^{2}(b)}{p_{K}^{2}}\right]=\sum_{k} \frac{\mathbb{E}^{Q}\left[Z_{k}^{2}(b)\right]}{p_{k}} \geq \sum_{k} \frac{\mathbb{P}\left\{n_{k-1}<\tau_{b} \leq n_{k}\right\}^{2}}{p_{k}},
$$

because of Jensen's inequality. To arrive at a contradiction, let us assume that both $\mathbb{E}^{Q}\left[Z^{2}(b)\right]$ and $\mathbb{E}^{Q}\left[\nu_{b}\right]$ are finite. Then,

$$
\begin{aligned}
\mathbb{E}^{Q}\left[Z^{2}(b)\right] \mathbb{E}^{Q}\left[\nu_{b}\right] & \geq\left(\sum_{k} \frac{\mathbb{P}\left\{n_{k-1}<\tau_{b} \leq n_{k}\right\}^{2}}{p_{k}}\right)\left(\sum_{k} p_{k} n_{k}\right) \\
& \geq\left(\sum_{k} \frac{\mathbb{P}\left\{n_{k-1}<\tau_{b} \leq n_{k}\right\}}{\sqrt{p_{k}}} \cdot \sqrt{p_{k} n_{k}}\right)^{2} \\
& =\left(\sum_{k} \sqrt{n_{k}} \mathbb{P}\left\{n_{k-1}<\tau_{b} \leq n_{k}\right\}\right)^{2} .
\end{aligned}
$$

where the penultimate step follows from Cauchy-Schwarz inequality. Then from Proposition 2 and Lemma 8, it is immediate that

$$
\begin{aligned}
\sum_{k} \sqrt{n_{k}} \mathbb{P}\left\{n_{k-1}<\tau_{b} \leq n_{k}\right\} & \geq(1-o(1)) \sum_{k} \sqrt{n_{k}} q_{k}(b) \\
& \geq(1-o(1)) \sum_{k} \sqrt{n_{k}} \int_{n_{k-1} \mu}^{n_{k} \mu} \bar{F}(b+u) d u \\
& \geq \frac{1-o(1)}{\sqrt{\mu}} \sum_{k} \int_{n_{k-1} \mu}^{n_{k} \mu} \sqrt{u} \bar{F}(b+u) d u \\
& =\frac{1-o(1)}{\sqrt{\mu}} \int_{0}^{\infty} \sqrt{u} \bar{F}(b+u) d u .
\end{aligned}
$$

Now it can be seen easily that the RHS is finite only when $\alpha \geq 1.5$, via the following change of variable and the subsequent integration of the resulting regularly varying tail:

$$
\begin{aligned}
\int_{0}^{\infty} \sqrt{u} \bar{F}(b+u) d u & =\int_{b}^{\infty} \sqrt{u-b} \bar{F}(u) d u \\
& \geq \int_{b^{2}}^{\infty} \sqrt{u} \cdot \sqrt{1-\frac{b}{u}} \bar{F}(u) d u \\
& \geq \sqrt{1-\frac{1}{b}} \int_{b^{2}}^{\infty} \sqrt{u} \bar{F}(u) d u
\end{aligned}
$$


which cannot be finite if $\alpha<1.5$, thus arriving at the desired contradiction. Therefore from (38), we conclude that we cannot have both the second moment of $Z(b)$ and the expected termination time $\mathbb{E}^{Q}\left[\nu_{b}\right]$ to be simultaneously finite if the tail index $\alpha<1.5$.

Proof of Theorem 6. The proof is similar to that of Theorem 4, and we provide only an outline of the steps involved. Since $Q(K=k)=p_{k}$,

$$
\begin{aligned}
\mathbb{E}^{Q}\left[Z^{1+\gamma}(b)\right] & =\mathbb{E}^{Q}\left[\frac{Z_{K}^{1+\gamma}(b)}{p_{K}^{1+\gamma}}\right]=\sum_{k} \frac{\mathbb{E}_{k}\left[Z_{k}^{1+\gamma}(b)\right]}{p_{k}^{1+\gamma}} p_{k} \\
& \leq \sum_{k}\left(\frac{\mathbb{E}_{k}\left[Z_{k}^{2}(b)\right]}{q_{k}^{2}(b)}\right)^{\frac{1+\gamma}{2}}\left(\frac{q_{k}(b)}{p_{k}}\right)^{\gamma} q_{k}(b)
\end{aligned}
$$

Now from Theorem 7 and (37), following the routine calculation in the proof of Theorem 4, we deduce that

$$
\begin{aligned}
\mathbb{E}^{Q}\left[Z^{1+\gamma}(b)\right] & \leq\left(c^{\frac{1+\gamma}{2}} r^{\beta \gamma}+o(1)\right)\left(\bar{G}_{I}^{(\beta)}(b)\right)^{\gamma} \sum_{k}\left(b+n_{k-1} \mu\right)^{\gamma(\beta-\alpha)} q_{k}(b) \\
& \leq\left(c^{\frac{1+\gamma}{2}} r^{\beta \gamma}+o(1)\right)\left(\bar{G}_{I}^{(\beta)}(b)\right)^{\gamma} \int_{b}^{\infty} u^{\gamma(\beta-\alpha)} \bar{F}(u) d u
\end{aligned}
$$

as $b \nearrow \infty$. Since $\beta$ is smaller than $\alpha+\gamma^{-1}(\alpha-1)$ as in the statement of Theorem 6 , the tail index of the integrand, $\alpha-\gamma(\beta-\alpha)>1$. Therefore we can apply Karamata's theorem to conclude that

$\mathbb{E}^{Q}\left[Z^{1+\gamma}(b)\right] \leq\left(c^{\frac{1+\gamma}{2}} r^{\beta \gamma}+o(1)\right)\left(\bar{G}_{I}^{(\beta)}(b)\right)^{\gamma} \frac{b^{\gamma(\beta-\alpha)+1}}{\alpha-\gamma(\beta-\alpha)-1} \bar{F}(b)$, as $b \nearrow \infty$.

Now observing that $(\alpha-1) \bar{F}_{I}(b) \sim b \bar{F}(b), b^{\beta-\alpha} \bar{G}_{I}^{(\beta)}(b) \sim \bar{F}_{I}(b)$, and $\mathbb{P}\left\{\tau_{b}<\right.$ $\infty\} \sim \mu^{-1} \bar{F}_{I}(b)$ as $b \nearrow \infty$, we have:

$$
\varlimsup_{b \rightarrow \infty} \frac{\mathbb{E}^{Q}\left[Z^{1+\gamma}(b)\right]}{\mathbb{P}\left\{\tau_{b}<\infty\right\}^{1+\gamma}} \leq \frac{\mu^{2}(\alpha-1) c^{\frac{1+\gamma}{2}} r^{\beta \gamma}+o(1)}{\alpha-\gamma(\beta-\alpha)-1}<\infty .
$$

Since $\beta$ is ensured to be larger than 2 , the same proof for $\mathbb{E}^{Q}\left[\nu_{b}\right]=O(b)$ goes through.

6. Simulation of $\tau_{\boldsymbol{b}}<\tau$. Let $X, X_{1}, X_{2}, \ldots$ be an iid collection of random variables satisfying the following assumption: 
Assumption 3 . The tail probabilities of $X$ are given by $\bar{F}(x):=\mathbb{P}\{X>$ $x\}=x^{-\alpha} L(x)$, for some slowly varying function $L(\cdot)$ and $\alpha>2$. Further, $\mu:=-\mathbb{E} X>0$.

As in the Sections 3 and 4 , let $S_{0}=0, S_{n}=X_{1}+\ldots+X_{n}$, for $n \geq 1$. Further, let $M_{n}=\max _{k \leq n} S_{k}, \tau=\inf \left\{n \geq 1: S_{n} \leq 0\right\}$ and $\tau_{b}=\inf \{n \geq 1$ : $\left.S_{n}>x\right\}$ for $b>0$. Our aim is to simulate the tail probabilities of busy cycle maximum $M_{\tau}$. In other words, we aim to simulate $\mathbb{P}\left\{M_{\tau}>b\right\}=\mathbb{P}\left\{\tau_{b}<\tau\right\}$ efficiently, as $b \nearrow \infty$. Under Assumption 3, it is well-known that (see, for example, Theorem 2.1 of [3])

$$
\mathbb{P}\left\{\tau_{b}<\tau\right\} \sim \mathbb{E} \tau \bar{F}(b), \text { as } b \nearrow \infty .
$$

As in the simulation of $\left\{S_{n}>b\right\}$, we partition the probability of interest into dominant and residual components as below:

$$
\mathbb{P}\left\{\tau_{b}<\tau\right\}=\mathbb{P}\left\{\tau_{b}<\tau, \max _{k \leq \tau_{b}} X_{k}>b\right\}+\mathbb{P}\left\{\tau_{b}<\tau, \max _{k \leq \tau_{b}} X_{k} \leq b\right\} .
$$

Since $S_{n}>0$ for all $n<\tau$, the first component has a simple representation:

$$
\begin{aligned}
\mathbb{P}\left\{\tau_{b}<\tau, \max _{k \leq \tau_{b}} X_{k}>b\right\} & =\mathbb{P}\left\{\tau_{b}<\tau, X_{\tau_{b}}>b\right\} \\
& =\sum_{n=1}^{\infty} \mathbb{P}\left\{S_{i} \in(0, b] \text { for } i=1, \ldots, n-1, X_{n}>b\right\} \\
& =\sum_{n=1}^{\infty} \mathbb{P}\left\{S_{i} \in(0, b] \text { for } i=1, \ldots, n-1\right\} \bar{F}(b) \\
& =\bar{F}(b) \sum_{n=1}^{\infty} \mathbb{P}\left\{\tau_{b} \wedge \tau>n-1\right\} \\
& =\mathbb{E}\left[\tau_{b} \wedge \tau\right] \bar{F}(b) .
\end{aligned}
$$

Therefore to estimate $\mathbb{P}\left\{\tau_{b}<\tau, \max _{k \leq \tau_{b}} X_{k}>b\right\}$, we draw samples of increments $X_{n}$ naively from the distribution $F(\cdot)$, and compute the following as the estimator:

$$
Z_{\text {dom }}(b):=\left(\tau_{b} \wedge \tau\right) \bar{F}(b) .
$$

Now it is straightforward to see that

$$
\begin{aligned}
\mathbb{E}\left[Z_{\text {dom }}\right] & =\mathbb{P}\left\{\tau_{b}<\tau, \max _{k \leq \tau_{b}} X_{k}>b\right\}, \\
\operatorname{Var}\left[Z_{\text {dom }}\right] & =\operatorname{Var}\left[\tau_{b} \wedge \tau\right] \bar{F}^{2}(b),
\end{aligned}
$$


and hence, due to (39) and monotone convergence,

$$
\varlimsup_{b \rightarrow \infty} \frac{\operatorname{Var}\left[Z_{\text {dom }}\right]}{\mathbb{P}\left\{\tau_{b}<\tau\right\}^{2}}=\varlimsup_{b \rightarrow \infty} \frac{\operatorname{Var}\left[\tau_{b} \wedge \tau\right]}{\mathbb{E}[\tau]^{2}}=\frac{\operatorname{Var}[\tau]}{\mathbb{E}[\tau]^{2}} .
$$

To estimate the residual probability $\mathbb{P}\left\{\tau_{b}<\tau\right.$, $\left.\max _{k \leq \tau_{b}} X_{k} \leq b\right\}$, we perform exponential twisting as in Section 3.2. Draw samples of $\left\{X_{n}: n \leq \tau_{b} \wedge \tau\right\}$ independently from $F_{\theta}(\cdot)$ given by:

$$
\frac{d F_{\theta}}{d F}(x)=\exp \left(\theta_{b} x-\Lambda_{b}\left(\theta_{b}\right)\right) \mathbf{1}(x \leq b),
$$

where

$$
\begin{aligned}
\Lambda_{b}(\theta) & :=\log \left(\int_{-\infty}^{b} \exp (\theta x) F(d x)\right) \text { for } \theta>0, \text { and } \\
\theta_{b} & :=-\frac{\log b \bar{F}(b)}{b} .
\end{aligned}
$$

Then the resulting estimator is given by

$$
Z_{\mathrm{res}}(b):=\exp \left(-\theta_{b} S_{\tau_{b}}+\tau_{b} \Lambda_{b}\left(\theta_{b}\right)\right) \mathbb{I}\left(\tau_{b}<\tau, \max _{k \leq \tau_{b}} X_{k} \leq b\right) .
$$

For proving efficiency results of $Z_{\mathrm{res}}(b)$, we shall need the following results that are proved in the appendix.

Proposition 3. Under Assumption 3,

$$
\mathbb{P}\left\{\tau_{b}<\tau, \max _{k \leq \tau_{b}} X_{k} \leq b\right\}=O\left(\frac{\bar{F}(b)}{b}\right) \text {, as } b \nearrow \infty .
$$

Lemma 9. Under Assumption 3, we have that

$$
\varlimsup_{b \rightarrow \infty} \sup _{n \geq 1} \exp \left(n \Lambda_{b}\left(\theta_{b}\right)\right) \leq 1 \text {. }
$$

Let $\mathbb{P}_{\theta}(\cdot)$ and $\mathbb{E}_{\theta}[\cdot]$ denote the probability measure and the corresponding expectation operator when the increments $X_{n}$ are drawn independent from $F_{\theta}(\cdot)$. Since $S_{\tau_{b}}>b$, it follows from the definition of $\theta_{b}$ and (43) that

$$
\begin{aligned}
\mathbb{E}_{\theta}\left[Z_{\mathrm{res}}^{2}(b)\right]=\mathbb{E}\left[Z_{\mathrm{res}}(b)\right] & \leq \mathbb{E}\left[\exp \left(-\theta_{b} b\right) \exp \left(\tau_{b} \Lambda_{b}\left(\theta_{b}\right)\right) ; \tau_{b}<\tau, \max _{k \leq \tau_{b}} X_{k}>b\right] \\
& \leq b \bar{F}(b) \sup _{n \geq 1} \exp \left(n \Lambda_{b}\left(\theta_{b}\right)\right) \mathbb{P}\left\{\tau_{b}<\tau, \max _{k \leq \tau_{b}} X_{k}>b\right\} \\
& =O\left(\bar{F}^{2}(b)\right), \text { as } b \nearrow_{\infty},
\end{aligned}
$$


because of Lemma 9 and Proposition 3. Then due to (39), it is immediate that

$$
\frac{\mathbb{E}_{\theta}\left[Z_{\mathrm{res}}^{2}(b)\right]}{\mathbb{P}\left\{\tau_{b}<\tau\right\}^{2}}=O(1) \text {, as } b \nearrow \infty .
$$

THEOREM 8. If the realizations of the estimators $Z_{\mathrm{dom}}(b)$ and $Z_{\mathrm{res}}(b)$ are generated respectively from the measures $\mathbb{P}(\cdot)$ and $\mathbb{P}_{\theta}(\cdot)$, and if we let

$$
Z(b):=Z_{\mathrm{dom}}(b)+Z_{\mathrm{res}}(b),
$$

then under Assumption 3, the family of estimators $(Z(b): b>0)$ are strongly efficient for the estimation of $\mathbb{P}\left\{\tau_{b}<\tau\right\}$, as $b \nearrow \infty$; that is,

$$
\frac{\operatorname{Var}[Z(b)]}{\mathbb{P}\left\{\tau_{b}<\tau\right\}^{2}}=O(1) \text {, as } b \nearrow \infty \text {. }
$$

Proof. Since $Z_{\text {dom }}(b)$ and $Z_{\text {res }}(b)$ are generated independently,

$$
\operatorname{Var}[Z(b)]=\operatorname{Var}\left[Z_{\mathrm{dom}}(b)\right]+\operatorname{Var}\left[Z_{\mathrm{res}}(b)\right] .
$$

This observation, together with (41) and (44) proves the claim.

7. Numerical Experiments. In this section, we present the results of numerical simulation experiments performed on examples previously considered in literature, and compare them with the performance of our algorithms.

7.1. Example 1 - Estimation of $\mathbb{P}\left\{S_{n}>b\right\}$. Take $X=\Lambda R$, where $\mathbb{P}\{\Lambda>$ $x\}=1 \wedge x^{-4}, R \sim$ Laplace(1), and $\Lambda$ is independent of $R$. We use $N=10,000$ simulation runs to estimate $\mathbb{P}\left\{S_{n}>n\right\}$ for $n=100,500$ and 1000. In Table 1 , we compare the numerical estimates obtained by our simulation procedure with the true values of $\mathbb{P}\left\{S_{n}>n\right\}$ evaluated in [13] via inverse transform techniques; further, a comparison of performance of our methodology with Algorithms 1 and 2 in [13] (referred to as BL1 and BL2) has also been presented. From the columns CV, CV of BL1, and CV of BL2, it can be inferred that our state-independent simulation procedures yield estimators with substantially lower coefficient of variation throughout the range of values considered. The state-dependent algorithms in comparison have been proven to be strongly efficient. The numerical performance of our algorithms in Table 1 just reflects the vanishing relative error of the estimators (a notion stronger than strong efficiency), which has been verified in Theorem 1 . 
TABLE 1

Numerical result for Example 1 - here Std. error denotes the standard deviation of the estimator of $\mathbb{P}\left\{S_{n}>n\right\}$ based on 10,000 simulation runs; $C V$ denotes the empirically observed coefficient of variation

\begin{tabular}{lllllll}
\hline $\mathrm{n}$ & $\mathbb{P}\left\{S_{n}>n\right\}$ & $\begin{array}{l}\text { Estimate }(\hat{z}) \\
\text { for } \mathbb{P}\left\{S_{n}>n\right\}\end{array}$ & Std. error & CV of $\hat{z}$ & $\begin{array}{l}\text { CV of } \\
\text { BL1 }\end{array}$ & $\begin{array}{l}\text { CV of } \\
\text { BL2 }\end{array}$ \\
\hline 100 & $2.21 \times 10^{-5}$ & $2.17 \times 10^{-5}$ & $4.31 \times 10^{-7}$ & 1.97 & 10.3 & 4.7 \\
500 & $1.04 \times 10^{-7}$ & $1.05 \times 10^{-7}$ & $6.91 \times 10^{-10}$ & 0.66 & 1.0 & 4.1 \\
1000 & $1.25 \times 10^{-8}$ & $1.29 \times 10^{-8}$ & $6.91 \times 10^{-11}$ & 0.53 & 1.1 & 3.8 \\
\hline
\end{tabular}

7.2. Example 2 - Estimation of $\mathbb{P}\left\{\tau_{b}<\infty\right\}$. To facilitate comparison with existing methods, we use the following example from [10]: Consider an $\mathrm{M} / \mathrm{G} / 1$ queue with traffic intensity $\rho=0.5$ and Pareto service times having tail $\mathbb{P}\{V>t\}=(1+t)^{-2.5}$. The aim is to estimate the probability that this queue develops a waiting time $b$ in stationarity by equivalently estimating the level crossing probabilities $\mathbb{P}\left\{\tau_{b}<\infty\right\}$ of the associated negative drift random walk. For this example, we use the simulation procedures discussed in Section 4 and compare the results with that of the existing algorithms in literature in Table 2. While Algorithms AK (in [7]) and DLW (in [22]) restrict the arrivals to be Poisson, the schemes BGL, BG and BL referring to the algorithms, respectively, in $[11,10]$ and $[14]$ do not impose any such restriction.

In our implementation, $r$ has been chosen to be 2 to keep the expected termination time low, as suggested by Theorem 3 . The results reported in Table 2 correspond to the simulation estimates of $\mathbb{P}\left\{\tau_{b}<\infty\right\}$ for values of $b=10^{2}, 10^{3}$ and $10^{4}$ using $N=10,000$ simulation runs. From Table 2, it can be inferred that the error offered by the estimates of our simpler stateindependent procedure is much smaller when compared with other existing algorithms. Table 3 gives a comparison of coefficient of variation of the estimators empirically observed for different values of $r$, and a fixed $b=10^{3}$. It can be seen from Table 3 as well that choosing $r=2$ helps in keeping the relative error low.

8. Conclusion. In this paper we revisited the problem of efficient simulation of commonly encountered rare event probabilities associated with random walks having regularly varying heavy-tailed increments. These comprised the large deviations probability of a random walk exceeding large values as well as level crossing probabilities corresponding to negative-drift random walks. In the existing literature there are results that suggest that state-independent methods for such probabilities are difficult to design. Significant research over the last few years has resulted in sophisticated state- 
TABLE 2

Numerical result for Example 2 - here Std. error denotes the standard deviation of the estimator of $\mathbb{P}\left\{\tau_{b}<\infty\right\}$ based on 10,000 simulation runs; $C V$ denotes the empirically observed coefficient of variation

\begin{tabular}{llll}
\hline $\begin{array}{l}\text { Estimation } \\
\text { Std. error }\end{array}$ & $b=10^{2}$ & $b=10^{3}$ & $b=10^{4}$ \\
CV & & & \\
\hline & $9.75 \times 10^{-4}$ & $3.15 \times 10^{-5}$ & $9.98 \times 10^{-7}$ \\
Proposed & $4.11 \times 10^{-6}$ & $7.89 \times 10^{-8}$ & $1.39 \times 10^{-9}$ \\
method & 0.42 & 0.25 & 0.14 \\
& $1.20 \times 10^{-3}$ & $3.15 \times 10^{-5}$ & $9.98 \times 10^{-7}$ \\
AK & $1.48 \times 10^{-5}$ & $2.19 \times 10^{-7}$ & $6.95 \times 10^{-9}$ \\
& 1.23 & 0.70 & 0.70 \\
DLW & $1.05 \times 10^{-3}$ & $3.16 \times 10^{-5}$ & $9.91 \times 10^{-7}$ \\
& $5.20 \times 10^{-6}$ & $1.69 \times 10^{-7}$ & $2.99 \times 10^{-9}$ \\
BGL & 0.50 & 0.53 & 0.30 \\
& $1.02 \times 10^{-3}$ & $3.17 \times 10^{-5}$ & $1.13 \times 10^{-6}$ \\
BG & $3.84 \times 10^{-5}$ & $1.60 \times 10^{-6}$ & $7.28 \times 10^{-8}$ \\
& 3.76 & 5.05 & 6.44 \\
BL & $1.08 \times 10^{-3}$ & $3.15 \times 10^{-5}$ & $9.98 \times 10^{-7}$ \\
& $5.97 \times 10^{-6}$ & $9.73 \times 10^{-8}$ & $2.07 \times 10^{-9}$ \\
& 0.55 & 0.31 & 0.21 \\
\hline
\end{tabular}

TABLE 3

Comparison of relative errors for different choices of $r$ in Example 2 with $b=1000$; here Std. error denotes the standard deviation of the estimator of $\mathbb{P}\left\{\tau_{b}<\infty\right\}$ based on 10,000 simulation runs; $C V$ denotes the empirically observed coefficient of variation

\begin{tabular}{l|ccc}
\hline$r$ & Estimate & Std. error & CV \\
\hline 2 & $3.15 \times 10^{-5}$ & $7.89 \times 10^{-8}$ & 0.25 \\
10 & $3.16 \times 10^{-5}$ & $1.03 \times 10^{-7}$ & 0.33 \\
100 & $3.16 \times 10^{-5}$ & $1.55 \times 10^{-7}$ & 0.49 \\
\hline
\end{tabular}

dependent importance sampling techniques for estimating these probabilities. Our key contribution has been to challenge this view by showing that simple state-independent importance sampling methods, that are at least as efficient as the existing state-dependent methods, can indeed be devised to estimate these probabilities.

Our approach relied on partitioning the rare event of interest into elementary events that are amenable to straight forward state-independent importance sampling methods. We expect that this approach will generalize to more complex, multi-dimensional problems, and for similar problems involving Weibull-type sub-exponential tail distributions. 


\section{APPENDIX A: PROOFS OF CERTAIN PROBABILITY ESTIMATES}

In this section we present proofs of Propositions 1, 2, 3 and Lemma 5. These asymptotic results on certain probabilities of interest have been useful in efficiency analysis of our algorithms. Some of these involve error estimates that have not been studied in the literature, and are interesting in their own right.

Proof of Proposition 1. Let $M_{n}:=\max \left\{X_{1}, \ldots, X_{n}\right\}$ for $n \geq 1$. We first obtain a lower bound for $\mathbb{P}\left\{S_{n}>b, X_{n}>b, M_{n-1} \leq b\right\}$ :

$$
\begin{aligned}
\mathbb{P}\left\{S_{n}>b, X_{n}>b, M_{n-1} \leq b\right\} & \geq \mathbb{P}\left\{S_{n-1}>-b^{\gamma}, M_{n-1} \leq b, X_{n}>b+b^{\gamma}\right\} \\
& =\mathbb{P}\left\{S_{n-1}>-b^{\gamma}, M_{n-1} \leq b\right\} \bar{F}\left(b+b^{\gamma}\right)
\end{aligned}
$$

for some $\gamma<1$ to be chosen later in the proof. Due to (8),

$$
\mathbb{P}\left\{M_{n-1}>b\right\} \sim(n-1) \bar{F}(b) \searrow 0
$$

uniformly for all $b>n^{\beta+\epsilon}$, as $n \nearrow \infty$. Here recall that $\beta:=(\alpha \wedge 2)^{-1}$. Similarly for $\gamma>\beta /(\beta+\epsilon)$, because of the convergence of $S_{n} / n^{\beta}$ to the stable distribution, we have $\mathbb{P}\left\{S_{n-1}<-b^{\gamma}\right\} \searrow 0$, uniformly for all $b>n^{\beta+\epsilon}$, as $n \nearrow \infty$. Therefore, it follows from union bound that,

$$
\mathbb{P}\left\{S_{n-1} \geq-b^{\gamma}, M_{n-1} \leq b\right\} \geq 1-o(1),
$$

uniformly for all $b>n^{\beta+\epsilon}$, as $n \nearrow \infty$. Since $\gamma<1$,

$$
\frac{\bar{F}\left(b+b^{\gamma}\right)}{\bar{F}(b)} \geq 1-o(1)
$$

because of (6). Combining these observations with (46), it follows that

$$
\mathbb{P}\left\{S_{n}>b, X_{n}>b, M_{n-1} \leq b\right\} \geq(1-o(1)) \bar{F}(b)
$$

uniformly for all $b>n^{\beta+\epsilon}$, as $n \nearrow \infty$.

Since $\mathbb{P}\left(A_{\text {res }}(n, b)\right)=\mathbb{P}\left\{S_{n}>b\right\}-\mathbb{P}\left\{S_{n}>b, M_{n}>b\right\}$,

$$
\begin{aligned}
\mathbb{P}\left(A_{\text {res }}(n, b)\right) & \leq \mathbb{P}\left\{S_{n}>b\right\}-\sum_{j=1}^{n} \mathbb{P}\left\{S_{n}>b, X_{j}>b, \max _{i \neq j, i \leq n} X_{i} \leq b\right\} \\
& =\mathbb{P}\left\{S_{n}>b\right\}-n \mathbb{P}\left\{S_{n}>b, X_{n}>b, M_{n-1} \leq b\right\} \\
& \leq(1+o(1)) n \bar{F}(b)-(1-o(1)) n \bar{F}(b)=o(n \bar{F}(b)),
\end{aligned}
$$

where the last inequality follows from (7) and (46). 
Proof of Proposition 2. The upper bound follows simply by applying union bound as below:

$$
\begin{aligned}
\mathbb{P}\left\{n_{k-1}<\tau_{b} \leq n_{k}, A_{k}\right\} & \leq \mathbb{P}\left\{\bigcup_{j=n_{k-1}+1}^{n_{k}}\left\{X_{j}>b+j \mu\right\}\right\} \\
& \leq \sum_{j=n_{k-1}+1}^{n_{k}} \bar{F}(b+j \mu)=q_{k}(b) .
\end{aligned}
$$

For obtaining a lower bound, see that

$$
\mathbb{P}\left\{n_{k-1}<\tau_{b} \leq n_{k}, A_{k}\right\}=\sum_{j=n_{k-1}+1}^{n_{k}} \mathbb{P}\left\{\tau_{b}=j, A_{k}\right\}
$$

is bounded from below by

$$
\sum_{j=n_{k-1}+1}^{n_{k}} \mathbb{P}\left\{\tau_{b}=j, S_{i}>-(b+i \mu)^{\gamma} \text { for all } i<j, X_{j}>b+j \mu+(b+j \mu)^{\gamma}\right\},
$$

for some $\gamma<1$ to be chosen later in this proof. Let $M_{n}:=\max _{k \leq n}\left(S_{k}-\right.$ $k \mu)$ and $M:=\sup _{k}\left(S_{k}-k \mu\right)$. Then $\mathbb{P}\left\{n_{k-1}<\tau_{b} \leq n_{k}, A_{k}\right\}$ is lower bounded by

$$
\begin{aligned}
& \sum_{j=n_{k-1}+1}^{n_{k}} \mathbb{P}\left\{M_{j-1} \leq b, S_{i}>-(b+i \mu)^{\gamma} \text { for all } i<j, X_{j}>b+j \mu+(b+j \mu)^{\gamma}\right\} \\
& \quad=\sum_{j=n_{k-1}+1}^{n_{k}} \mathbb{P}\left\{M_{j-1} \leq b, S_{i}>-(b+i \mu)^{\gamma} \text { for all } i<j\right\} \bar{F}\left(b+j \mu+(b+j \mu)^{\gamma}\right)
\end{aligned}
$$

$$
\geq \mathbb{P}\left\{M \leq b, \min _{i<n_{k}} S_{i}>-\left(b+n_{k-1} \mu\right)^{\gamma}\right\} \sum_{j=n_{k-1}+1}^{n_{k}} \bar{F}\left(b+j \mu+(b+j \mu)^{\gamma}\right)
$$

From (16) we have that $\mathbb{P}\{M>b\} \sim \mu^{-1} \bar{F}_{I}(b)$ as $b \nearrow \infty$. Recall that $\beta=(\alpha \wedge 2)^{-1}$. If $\gamma>\beta$, then under the lighter left tail assumption formally stated in Assumption 1,

$$
\mathbb{P}\left\{\min _{i<n_{k}} S_{i}<-\left(b+n_{k-1} \mu\right)^{\gamma}\right\}=O\left(n_{k}\left(b+n_{k-1} \mu\right)^{-\frac{\gamma}{\beta}+o(1)}\right), \text { as } b \nearrow \infty .
$$

This follows from the well-known large deviation asymptotic that

$$
\mathbb{P}\left\{\max _{i \leq n} S_{i}>x\right\} \sim n \bar{F}(x)
$$


uniformly for $x>n^{\beta+\epsilon}$; this can be found, for example, in Theorem 2.2 of [17] and Theorem 5 of [15]. Therefore, by union bound,

$$
\begin{aligned}
\mathbb{P}\{M & \left.\leq b, \min _{i<n_{k}} S_{i}>-\left(b+n_{k-1} \mu\right)^{\gamma}\right\} \\
& \geq 1-\mathbb{P}\{M>b\}-\mathbb{P}\left\{\min _{i<n_{k}} S_{i}<-\left(b+n_{k-1} \mu\right)^{\gamma}\right\} \\
& \geq 1-\bar{F}_{I}(b)(1-o(1))-O\left(n_{k}\left(b+n_{k-1} \mu\right)^{-\frac{\gamma}{\beta}+o(1)}\right),
\end{aligned}
$$

as $b \nearrow \infty$. Further because of (6),

$$
\begin{aligned}
\sum_{j=n_{k-1}+1}^{n_{k}} \bar{F}\left(b+j \mu+(b+j \mu)^{\gamma}\right) & \geq \sum_{j=n_{k-1}+1}^{n_{k}}\left(1+\frac{(b+j \mu)^{\gamma}}{b+j \mu}\right)^{-\alpha+o(1)} \bar{F}(b+j \mu) \\
& \geq\left(1-\frac{c}{\left(b+n_{k-1} \mu\right)^{1-\gamma}}\right) \sum_{j=n_{k-1}+1}^{n_{k}} \bar{F}(b+j \mu)
\end{aligned}
$$

for some positive constant $c$. If we choose $\gamma=(\alpha+1) / 2 \alpha$, then

$$
\sum_{j=n_{k-1}+1}^{n_{k}} \bar{F}\left(b+j \mu+(b+j \mu)^{\gamma}\right) \geq\left(1-\frac{c}{\left(b+n_{k-1} \mu\right)^{\frac{\alpha-1}{2 \alpha}}}\right) q_{k}(b) .
$$

Combining this with (48) and (49), we see that

$$
\mathbb{P}\left\{n_{k-1}<\tau_{b} \leq n_{k}, A_{k}\right\} \geq\left(1-\frac{c+o(1)}{b^{\frac{\alpha-1}{2 \alpha}}}\right) q_{k}(b),
$$

as $b \nearrow \infty$. Along with (47), we have that

$$
\sup _{k}\left|\frac{\mathbb{P}\left\{n_{k-1}<\tau_{b} \leq n_{k}, A_{k}\right\}}{q_{k}(b)}-1\right|=O\left(b^{-\frac{\alpha-1}{2 \alpha}}\right), \text { as } b \nearrow \infty .
$$

Proof of Lemma 5. Recall that $n_{k}=r n_{k-1}$ for some constant $r$. Therefore, for any $k \geq 1$,

$$
1 \leq \frac{\sum_{j=1}^{n_{k}} \bar{F}(b+j \mu)}{\sum_{j=1}^{n_{k-1}} \bar{F}(b+j \mu)}=1+\frac{\sum_{j=n_{k-1}+1}^{n_{k}} \bar{F}(b+j \mu)}{\sum_{j=1}^{n_{k-1}} \bar{F}(b+j \mu)} \leq 1+\frac{n_{k-1} \bar{F}\left(b+n_{k-1} \mu\right)}{n_{k-1} \bar{F}\left(b+n_{k-1} \mu\right)}=2 .
$$


When $\operatorname{Var}[X]<\infty$, see from (17) and Proposition 2 that $\mathbb{P}\left\{n_{k-1}<\tau_{b} \leq\right.$ $\left.n_{k}, \bar{A}_{k}\right\}$ equals

$$
\begin{aligned}
& \mathbb{P}\left\{\tau_{b} \leq n_{k}\right\}-\mathbb{P}\left\{\tau_{b} \leq n_{k-1}\right\}-\mathbb{P}\left\{n_{k-1}<\tau_{b} \leq n_{k}, A_{k}\right\} \\
& =\sum_{j=1}^{n_{k}} \bar{F}(b+j \mu)\left(1+O\left(\frac{1}{b}\right)\right)+o\left(\sqrt{n_{k} \wedge b} \bar{F}(b)\right) \\
& \quad-\sum_{j=1}^{n_{k-1}} \bar{F}(b+j \mu)\left(1+O\left(\frac{1}{b}\right)\right)-\left(1-O\left(b^{-\frac{\alpha-1}{2 \alpha}}\right)\right) \sum_{j=n_{k-1}+1}^{n_{k}} \bar{F}(b+j \mu),
\end{aligned}
$$

$$
=\sum_{j=1}^{n_{k}} \bar{F}(b+j \mu)\left(O\left(b^{-\frac{\alpha-1}{2 \alpha}}\right)\right)+o\left(\sqrt{n_{k} \wedge b} \bar{F}(b)\right)
$$

as $b \nearrow \infty$. Similarly when $\operatorname{Var}[X]=\infty$, for $k$ such that $n_{k} \bar{F}(b)=o(1)$, see from (18) and Proposition 2 that $\mathbb{P}\left\{n_{k-1}<\tau_{b} \leq n_{k}, \bar{A}_{k}\right\}$ equals

$$
\begin{array}{r}
\sum_{j=1}^{n_{k}} \bar{F}(b+j \mu)\left(1+O\left(\frac{n_{k}^{\frac{1}{\alpha}+\epsilon}}{b}\right)\right) \\
-\sum_{j=1}^{n_{k-1}} \bar{F}(b+j \mu)\left(1+O\left(\frac{n_{k-1}^{\frac{1}{\alpha}+\epsilon}}{b}\right)\right) \\
-\left(1-O\left(b^{-\frac{\alpha-1}{2 \alpha}}\right)\right) \sum_{j=n_{k-1}+1}^{n_{k}} \bar{F}(b+j \mu) \\
=\sum_{j=1}^{n_{k}} \bar{F}(b+j \mu)\left(O\left(\frac{n_{k}^{\frac{1}{\alpha}+\epsilon}}{b}\right)+O\left(b^{-\frac{\alpha-1}{2 \alpha}}\right)\right)
\end{array}
$$

for every $\epsilon>0$. Since $n_{k}=r n_{k-1}$ for some constant $r$, it follows from (6) that for small enough $\epsilon$ and suitably chosen $\eta>1$,

$$
\begin{gathered}
\sup _{k: n_{k}<b^{\eta}} \frac{b^{-\frac{\alpha-1}{2 \alpha}} \sum_{j=1}^{n_{k}} \bar{F}(b+j \mu)}{q_{k}(b)} \leq \sup _{k: n_{k}<b^{\eta}} \frac{b^{-\frac{\alpha-1}{2 \alpha}} n_{k} \bar{F}(b)}{n_{k-1} \bar{F}\left(b+n_{k} \mu\right)}=o(1), \\
\sup _{k: n_{k}<b^{\eta}} \frac{n_{k}^{\frac{1}{\alpha}+\epsilon}}{b} \frac{\sum_{j=1}^{n_{k}} \bar{F}(b+j \mu)}{q_{k}(b)} \leq \sup _{k: n_{k}<b^{\eta}} \frac{n_{k}^{\frac{1}{\alpha}+\epsilon}}{b} \frac{n_{k} \bar{F}(b)}{n_{k-1} \bar{F}\left(b+n_{k} \mu\right)}=o(1), \text { and } \\
\sup _{k: n_{k}<b^{\eta}} \frac{\sqrt{n_{k} \wedge b} \bar{F}(b)}{\sum_{j=1}^{n_{k}} \bar{F}(b+j \mu)} \leq \sup _{k: n_{k}<b^{\eta}} \frac{\sqrt{n_{k} \wedge b} \bar{F}(b)}{n_{k} \bar{F}\left(b+n_{k} \mu\right)}=o(1),
\end{gathered}
$$

as $b \nearrow \infty$. Therefore from (51) and (52), for some $\eta>1$,

$$
\sup _{k: n_{k}<b^{\eta}} \frac{\mathbb{P}\left\{n_{k-1}<\tau_{b} \leq n_{k}, \bar{A}_{k}\right\}}{q_{k}(b)}=o(1), \text { as } b \nearrow \infty .
$$


For $k$ such that $n_{k}>b^{\eta}$, we obtain a loose bound that suffices for our purposes:

$$
\begin{aligned}
\mathbb{P}\left\{n_{k-1}<\tau_{b} \leq n_{k}\right\}= & \mathbb{P}\left\{n_{k-1}<\tau_{b} \leq n_{k}, S_{n_{k-1}}>\frac{b+n_{k-1} \mu}{2}\right\} \\
& +\mathbb{P}\left\{n_{k-1}<\tau_{b} \leq n_{k}, S_{n_{k-1}} \leq \frac{b+n_{k-1} \mu}{2}\right\} \\
\leq & \mathbb{P}\left\{S_{n_{k-1}}>\frac{b+n_{k-1} \mu}{2}\right\}+\mathbb{P}\left\{\tau_{\frac{\tau_{n-n_{k-1} \mu}}{2}}<\infty\right\} \\
\leq & (1+\epsilon) n_{k-1} \bar{F}\left(\frac{b+n_{k-1} \mu}{2}\right)+\frac{(1+\epsilon)}{\mu} \bar{F}_{I}\left(\frac{b+n_{k-1} \mu}{2}\right),
\end{aligned}
$$

for all $k, b$ large enough. While the final inequality is due to the asymptotics (7) and (16), the second term in the penultimate step follows by observing that whenever the event $\left\{n_{k-1}<\tau_{b} \leq n_{k}, S_{n_{k-1}} \leq\left(b+n_{k-1} \mu\right) / 2\right\}$ happens, the random walk $\left(Z_{n}: n \geq 0\right)$ defined by

$$
Z_{n}:=S_{n+n_{k-1}}-S_{n_{k-1}}-n \mu
$$

crosses the level $\left(b+n_{k-1} \mu\right) / 2$ at some finite $n \leq n_{k}-n_{k-1}$. Here recall that $\tau_{x}:=\inf \left\{k \geq 1: S_{k}>x+k \mu\right\}$.

Further, since $n_{k}=r n_{k-1}$ for some constant $r$, from (5) and (6), we have that

$$
\sup _{k: n_{k}>b^{\eta}} \frac{n_{k-1} \bar{F}\left(\frac{b+n_{k-1} \mu}{2}\right)}{q_{k}(b)}<\infty \text { and } \sup _{k: n_{k}>b^{\eta}} \frac{\bar{F}_{I}\left(\frac{b+n_{k-1} \mu}{2}\right)}{q_{k}(b)}<\infty .
$$

Therefore from (54),

$$
\sup _{k: n_{k}>b^{\eta}} \frac{\mathbb{P}\left\{n_{k-1}<\tau_{b} \leq n_{k}, \bar{A}_{k}\right\}}{q_{k}(b)}<\infty,
$$

which along with (53) establishes the claim.

Proof of Proposition 3. Consider

$$
\begin{aligned}
P_{1} & :=\mathbb{P}\left\{\tau_{b}<\tau, \max _{k \leq \tau_{b}} X_{k} \leq b, S_{\tau_{b}-1}<\frac{b}{2}\right\} \\
& \leq \mathbb{P}\left\{S_{n} \in\left(0, \frac{b}{2}\right), S_{n+1}>b, X_{n+1}<b \text { for some } n<\tau\right\} \\
& \leq \mathbb{E}\left[\sum_{n=0}^{\tau-1} \mathbb{I}\left(S_{n} \in\left(0, \frac{b}{2}\right), S_{n+1}>b, X_{n+1}<b\right)\right] \\
& \leq \mathbb{E}\left[\sum_{n=0}^{\tau-1} \mathbb{I}\left(S_{n} \in\left(0, \frac{b}{2}\right)\right) \mathbb{P}\left\{b-S_{n}<X<b\right\}\right]
\end{aligned}
$$


Let $\pi(B)=\mathbb{P}\left\{\sup _{n} S_{n} \in B\right\}$. Then according to the regenerative ratio representation,

$\frac{1}{\mathbb{E} \tau} \mathbb{E}\left[\sum_{n=0}^{\tau-1} \mathbb{I}\left(S_{n} \in\left(0, \frac{b}{2}\right)\right) \mathbb{P}\left\{b-S_{n}<X<b\right\}\right]=\int_{0}^{\frac{b}{2}} \mathbb{P}\{b-u<X<b\} \pi(d u)$.

Therefore,

$$
\begin{aligned}
P_{1} & \leq \mathbb{E} \tau \int_{0}^{\frac{b}{2}}(\bar{F}(b-u)-\bar{F}(b)) \pi(d u) \\
& =\mathbb{E} \tau \bar{F}(b) \int_{0}^{\frac{b}{2}}\left(\frac{\bar{F}(b-u)}{\bar{F}(b)}-1\right) \pi(d u) .
\end{aligned}
$$

From Potter's bounds (see (6)), we have that for all $u<\frac{b}{2}$,

$$
\frac{\bar{F}(b-u)}{\bar{F}(b)} \leq\left(1-\frac{u}{b}\right)^{-\alpha-\delta} \leq 1+(\alpha+\delta) 2^{\alpha+\delta+1} \frac{u}{b},
$$

for any $\delta>0$ and all $b$ large enough. The last inequality follows from Taylor's theorem. Hence

$$
P_{1} \leq(\alpha+\delta) 2^{\alpha+\delta+1} \mathbb{E} \tau \frac{\bar{F}(b)}{b} \int_{0}^{\frac{b}{2}} u \pi(d u) .
$$

Recall that $\pi((x, \infty)) \sim \int_{x}^{\infty} \bar{F}(u) d u$. Since $\alpha>2, \int_{0}^{\infty} u \pi(d u)<\infty$. Therefore,

$$
P_{1}=O\left(\frac{\bar{F}(b)}{b}\right), \text { as } b \nearrow \infty .
$$

Now consider the complementary event $\left\{\tau_{b}<\tau, \max _{k \leq \tau_{b}} X_{k} \leq b, S_{\tau_{b}-1}>b / 2\right\}$ :

$$
\begin{aligned}
P_{2} & :=\mathbb{P}\left\{\tau_{b}<\tau, \max _{k \leq \tau_{b}} X_{k} \leq b, S_{\tau_{b}-1}>\frac{b}{2}\right\} \\
& =\sum_{n=1}^{\infty} \mathbb{P}\left\{\tau>n, \max _{k \leq n} X_{k} \leq b, S_{n-1}>\frac{b}{2}, \tau_{b}=n\right\} \\
& =\sum_{n=1}^{\infty} \mathbb{P}\left\{\tau>n, \max _{k \leq n} X_{k} \leq b, S_{n-1}>\frac{b}{2}, M_{n-1} \leq b, S_{n}>b\right\} \\
& =\sum_{n=1}^{\infty} \int_{\frac{b}{2}}^{b} \mathbb{P}\left\{\tau>n, \max _{k \leq n} X_{k} \leq b, S_{n-1} \in d y, M_{n-1} \leq b, S_{n}>b\right\} \\
& \leq \sum_{n=1}^{\infty} \int_{\frac{b}{2}}^{b} \mathbb{P}\left\{\tau>n, S_{n-1} \in d y, M_{n-1} \leq b\right\} F((b-y, b]),
\end{aligned}
$$


where the notation $F((x, y])$ stands for $\mathbb{P}\{x<X \leq y\}$. Consider the taboo renewal function $H_{x}(\cdot)$ defined below:

$$
H_{x}(B):=\sum_{n=0}^{\infty} \mathbb{P}\left\{\tau>n, M_{n} \leq x, S_{n} \in B\right\} .
$$

Then it is immediate that

$$
P_{2} \leq \int_{\frac{b}{2}}^{b} H_{b}(d y) F((b-y, b]) .
$$

From Theorem 2 of [20], given $\epsilon>0$, we have a $y_{0}$ large enough such that, for all $x$ and $y$ with $y \in\left(y_{0}, x-y_{0}\right)$,

$$
(1-\epsilon) \frac{\mathbb{E} \tau}{\mu} F((y, x]) d y \leq H_{x}((y, y+d y)) \leq(1+\epsilon) \frac{\mathbb{E} \tau}{\mu} F((y, x]) d y .
$$

Therefore, for a fixed $\epsilon>0$, we have

$$
H_{b+c}((y, y+d y)) \leq(1+\epsilon) \frac{\mathbb{E} \tau}{\mu} F((y, b+c]) d y
$$

in the interval $(b / 2, b)$, for some constant $c$ and all $b$ large enough. Since $H_{b}(\cdot) \leq H_{b+c}(\cdot)$,

$$
\begin{aligned}
P_{2} & \leq(1+\epsilon) \frac{\mathbb{E} \tau}{\mu} \int_{\frac{b}{2}}^{b} F((y, b+c]) F((b-y, b]) d y \\
& =(1+\epsilon) \frac{\mathbb{E} \tau}{\mu} \int_{0}^{\frac{b}{2}} F((b-y, b+c]) F((y, b]) d y \\
& \leq(1+\epsilon) \frac{\mathbb{E} \tau}{\mu}\left(\bar{F}(b) \int_{0}^{\frac{b}{2}} \frac{F((b-y, b])}{\bar{F}(b)} F((y, b]) d y+\int_{0}^{\frac{b}{2}} F((b, b+c]) F((y, b]) d y\right)
\end{aligned}
$$

For a fixed $\delta>0$, it follows from (6) that

$$
\begin{aligned}
\frac{F((b-y, b])}{\bar{F}(b)} & =\bar{F}(b)\left(\frac{\bar{F}(b-y)}{\bar{F}(b)}-1\right) \\
& \leq \bar{F}(b)\left(\left(1-\frac{y}{b}\right)^{-\alpha-\delta}-1\right) \\
& \leq(\alpha+\delta) 2^{\alpha+\delta+1} \frac{y}{b} \bar{F}(b)
\end{aligned}
$$


for all $y<b / 2$ and $b$ large enough. The last inequality is a consequence of Taylor's theorem. Then,

$$
P_{2} \leq(1+\epsilon) \frac{\mathbb{E} \tau}{\mu}\left((\alpha+\delta) 2^{\alpha+\delta+1} \frac{\bar{F}(b)}{b} \int_{0}^{\frac{b}{2}} y \bar{F}(y) d y+F((b, b+c]) \int_{0}^{\frac{b}{2}} F((y, b]) d y\right) .
$$

Since $\bar{F}(\cdot)$ is regularly varying, given $\gamma>0$ it follows from (6) that for all $b$ large enough,

$$
\begin{aligned}
F((b, b+c]) & =\bar{F}(b)\left(1-\frac{\bar{F}(b+c)}{\bar{F}(b)}\right) \\
& \leq \bar{F}(b)\left(1-\left(1+\frac{c}{b}\right)^{-\alpha-\gamma}\right) \\
& \leq \bar{F}(b)\left((\alpha+\gamma) \frac{c}{b}\right) .
\end{aligned}
$$

Therefore

$$
F((b, b+c])=O\left(\frac{\bar{F}(b)}{b}\right), \text { as } b \nearrow \infty .
$$

Further, when $\alpha>2$,

$$
\int_{0}^{\infty} \bar{F}(u) d u<\infty \text { and } \int_{0}^{\infty} u \bar{F}(u) d u<\infty .
$$

Using these in (56), we obtain that for tails with regularly varying index $\alpha>2$,

$$
P_{2}=O\left(\frac{\bar{F}(b)}{b}\right)
$$

as $b \nearrow \infty$. Therefore, from (55) and (57), we obtain

$$
\mathbb{P}\left\{\tau_{b}<\tau, \max _{k \leq \tau_{b}} X_{k}<b\right\}=P_{1}+P_{2}=O\left(\frac{\bar{F}(b)}{b}\right),
$$

as $b \nearrow \infty$. This proves the claim.

\section{APPENDIX B: PROOFS OF OTHER LEMMAS}

Here we present proofs of Lemmas 1, 3, 4 and 9. To prove Lemmas 1, 3 and 9, we need Lemmas 10 and 11, which are stated and proved below. The proof of Lemma 10 follows the lines of Theorem 4.1.2 of [16], where bounds for similar integrals have been derived. 
Lemma 10. For any pair of sequences $\left\{x_{n}\right\},\left\{\phi_{n}\right\}$ satisfying $x_{n} \nearrow \infty$ and $\phi_{n} x_{n} \nearrow \infty$, the integral,

$$
\int_{-\infty}^{x_{n}} e^{\phi_{n} x} F(d x) \leq 1+c \phi_{n}^{\kappa}+e^{2 \alpha} \bar{F}\left(\frac{2 \alpha}{\phi_{n}}\right)+e^{\phi_{n} x_{n}} \bar{F}\left(x_{n}\right)(1+o(1)),
$$

as $n \nearrow \infty$, for any $1<\kappa<\alpha \wedge 2$, and some constant $c$ which does not depend on $n$ and $b$.

Proof. We split the region of integration into $\left(-\infty, \gamma / \phi_{n}\right]$ and $\left(\gamma / \phi_{n}, x_{n}\right]$ for some constant $\gamma>0$; the partition is such that the integrand stays bounded in the former region.

Let $I_{1}:=\int_{-\infty}^{\gamma / \phi_{n}} e^{\phi_{n} x} F(d x)$ and $I_{2}:=\int_{\gamma / \phi_{n}}^{x_{n}} e^{\phi_{n} x} F(d x)$.

For any $\kappa \in(1,2]$ and $y>0$, it is easily verified that

$$
e^{x} \leq 1+x+|x|^{\kappa} e^{y}, \quad x \in(-\infty, y] .
$$

Therefore,

$$
\begin{aligned}
I_{1} & \leq \int_{-\infty}^{\gamma / \phi_{n}}\left(1+\phi_{n} x+\phi_{n}^{\kappa}|x|^{\kappa} \exp \left(\phi_{n} \cdot \gamma / \phi_{n}\right)\right) F(d x) \\
& \leq \int_{-\infty}^{\gamma / \phi_{n}} F(d x)+\phi_{n} \int_{-\infty}^{\gamma / \phi_{n}} x F(d x)+\phi_{n}^{\kappa} e^{\gamma} \int_{-\infty}^{\gamma / \phi_{n}}|x|^{\kappa} F(d x) \\
& \leq \int_{-\infty}^{\infty} F(d x)+\phi_{n} \int_{-\infty}^{\infty} x F(d x)+\phi_{n}^{\kappa} e^{\gamma} \int_{-\infty}^{\infty}|x|^{\kappa} F(d x) \\
& =1+c \phi_{n}^{\kappa},
\end{aligned}
$$

where $c:=e^{\gamma} \int_{-\infty}^{\infty}|x|^{\kappa} F(d x)<\infty$ because $\mathbb{E}|X|^{\kappa}<\infty$; this follows because $\kappa<\alpha$ and from Assumption 1. We have also used $\mathbb{E} X=0$ to arrive at (58). Integrating by parts for the second integral $I_{2}$ :

$$
\begin{aligned}
I_{2} & =-\int_{\gamma / \phi_{n}}^{x_{n}} e^{\phi_{n} x} \bar{F}(d x) \\
& =e^{\phi_{n} \gamma / \phi_{n}} \bar{F}\left(\frac{\gamma}{\phi_{n}}\right)-e^{\phi_{n} x_{n}} \bar{F}\left(x_{n}\right)+\phi_{n} \int_{\gamma / \phi_{n}}^{x_{n}} e^{\phi_{n} x} \bar{F}(x) d x \\
& \leq e^{\gamma} \bar{F}\left(\frac{\gamma}{\phi_{n}}\right)+I_{2}^{\prime},
\end{aligned}
$$

where, $I_{2}^{\prime}:=\phi_{n} \int_{\gamma / \phi_{n}}^{x_{n}} e^{\phi_{n} x} \bar{F}(x) d x$. Now the change of variable $u=\phi_{n}\left(x_{n}-x\right)$ 
results in:

$$
\begin{aligned}
I_{2}^{\prime} & =e^{\phi_{n} x_{n}} \int_{0}^{\phi_{n} x_{n}-\gamma} e^{-u} \bar{F}\left(x_{n}-\frac{u}{\phi_{n}}\right) d u \\
& =e^{\phi_{n} x_{n}} \bar{F}\left(x_{n}\right) \int_{0}^{\phi_{n} x_{n}-\gamma} e^{-u} g_{n}(u) d u
\end{aligned}
$$

where,

$$
g_{n}(u):=\frac{\bar{F}\left(x_{n}-\frac{u}{\phi_{n}}\right)}{\bar{F}\left(x_{n}\right)}=\frac{\bar{F}\left(x_{n}\left(1-\frac{u}{\phi_{n} x_{n}}\right)\right)}{\bar{F}\left(x_{n}\right)} .
$$

Since $L(\cdot)$ is slowly varying and $\phi_{n} x_{n} \rightarrow \infty$, given any $\delta>0$, it follows from (6) that,

$$
(1-\delta)\left(1-\frac{u}{\phi_{n} x_{n}}\right)^{-\alpha+\delta} \leq g_{n}(u) \leq(1+\delta)\left(1-\frac{u}{\phi_{n} x_{n}}\right)^{-\alpha-\delta} .
$$

for all $n$ large enough. So for any fixed $u$, we have $g_{n}(u) \rightarrow 1$ as $n \nearrow \infty$. Now fix $\delta=\frac{\alpha}{2}$. Then for $n$ large enough,

$$
g_{n}(u) \leq\left(1+\frac{\alpha}{2}\right)\left(1-\frac{u}{\phi_{n} x_{n}}\right)^{-\frac{3 \alpha}{2}} .
$$

Let $h(u)=\left(1-u / \phi_{n} x_{n}\right)^{-\frac{3 \alpha}{2}}$. Since $\log h(0)=0$ and $\frac{d}{d u}\left(\log (h(u)) \leq \frac{3 \alpha}{2 \gamma}\right.$ for $0 \leq u \leq \phi_{n} x_{n}-\gamma$, we have $h(u) \leq \exp (3 \alpha u / 2 \gamma)$ on the same interval. Therefore if we choose $\gamma=2 \alpha$, the integrand in $I_{2}^{\prime}$ is bounded for large enough $n$ by an integrable function as below:

$$
\begin{aligned}
\left|e^{-u} g_{n}(u) \mathbf{1}\left(0 \leq u \leq \phi_{n} x_{n}-\gamma\right)\right| & \leq\left|e^{-u}\left(1+\frac{\alpha}{2}\right) h(u) \mathbf{1}\left(0 \leq u \leq \phi_{n} x_{n}-\gamma\right)\right| \\
& \leq\left(1+\frac{\alpha}{2}\right) e^{-u+\frac{3 \alpha u}{2 \gamma}}=\left(1+\frac{\alpha}{2}\right) e^{-\frac{u}{4}}
\end{aligned}
$$

Applying dominated convergence theorem, we get

$$
\int_{0}^{\phi_{n} x_{n}-\gamma} e^{-u} g_{n}(u) d u \sim 1 \text { as } n \nearrow \infty .
$$

Since $\int_{-\infty}^{x_{n}} e^{\phi_{n} x} F(d x)=I_{1}+I_{2}$, combining this result with (58), (59) and (60), completes the proof.

Lemma 11. Given any $\epsilon>0$, uniformly for $b>n^{\beta+\epsilon}$, we have:

(a) $n \theta_{n, b}^{\kappa} \searrow 0$ for some $1<\kappa<\alpha \wedge 2$, and 
(b) $\bar{F}\left(2 \alpha / \theta_{n, b}\right)=o(1 / n)$, as $n \nearrow \infty$.

Proof. (a) We have $\bar{F}(x)=x^{-\alpha} L(x)$. Since $L(\cdot)$ is slowly varying, following (6) we have that $L(b)=b^{o(1)}$ as $b \nearrow \infty$. Further noting that $b>n^{\beta+\epsilon}$ helps us to write:

$$
n \theta_{n, b}^{\kappa}=\frac{n}{b^{\kappa}} \log ^{\kappa}\left(\frac{1}{n \bar{F}(b)}\right) \leq n^{1-\kappa(\beta+\epsilon)} \log ^{\kappa}\left(\frac{b^{\alpha}}{n L(b)}\right) .
$$

If we choose $\kappa \in\left((\beta+\epsilon)^{-1}, \alpha\right)$ then $\kappa(\beta+\epsilon)>1$ and subsequently $n \theta_{n, b}^{\kappa} \searrow 0$ as $n \nearrow \infty$, uniformly for $b>n^{\beta+\epsilon}$.

(b) We have $\theta_{n, b}:=-\log (n \bar{F}(b)) / b$. Therefore,

$$
n \bar{F}\left(\frac{2 \alpha}{\theta_{n}}\right)=n \bar{F}(b) \frac{\bar{F}\left(\frac{2 \alpha b}{-\log (n \bar{F}(b))}\right)}{\bar{F}(b)} .
$$

Since $\bar{F}(\cdot)$ is regularly varying, given any $\delta>0$, it follows from (6) that

$$
\frac{\bar{F}\left(\frac{2 \alpha b}{-\log (n \bar{F}(b))}\right)}{\bar{F}(b)} \leq\left(\frac{-\log (n \bar{F}(b))}{2 \alpha}\right)^{\alpha+\delta},
$$

for $n$ large enough. Therefore,

$$
n \bar{F}\left(\frac{2 \alpha}{\theta_{n}}\right) \leq n \frac{L(b)}{b^{\alpha}}\left(\frac{-\log (n \bar{F}(b))}{2 \alpha}\right)^{\alpha+\delta}=o(1),
$$

uniformly for $b>n^{\beta+\epsilon}$ as $n \nearrow \infty$. Here the convergence to 0 is justified because $\alpha>1$ and $b>n^{\beta+\epsilon}$.

Proof of Lemma 1. From the definition of $\Lambda_{b}(\cdot)$ and Lemma 10, we have:

$$
\begin{aligned}
\exp \left(\Lambda_{b}\left(\theta_{n, b}\right)\right) & =\int_{-\infty}^{b} \exp \left(\theta_{n, b} x\right) F(d x) \\
& \leq 1+c \theta_{n, b}^{\kappa}+e^{2 \alpha} \bar{F}\left(\frac{2 \alpha}{\theta_{n, b}}\right)+\exp \left(\theta_{n, b}\right) \bar{F}(b)(1+o(1))
\end{aligned}
$$

for $\kappa \in\left((\beta+\epsilon)^{-1}, \alpha\right)$. Usage of Lemma 10 is justified because $b \theta_{n, b}=$ $-\log (n \bar{F}(b)) \nearrow \infty$. The last term,

$$
\exp \left(\theta_{n, b} b\right) \bar{F}(b)=\frac{1}{n \bar{F}(b)} \bar{F}(b)=\frac{1}{n} .
$$


From Lemma 11, we have $n \theta_{n, b}^{\kappa}=o(1)$ and $\bar{F}\left(2 \alpha / \theta_{n, b}\right)=o(1 / n)$, uniformly for $b>n^{\beta+\epsilon}$. Therefore,

$$
\exp \left(\Lambda_{b}\left(\theta_{n}\right)\right) \leq 1+\frac{1}{n}(1+o(1)), \text { as } n \nearrow \infty .
$$

Proof of Lemma 3. Consider $\theta: \mathbb{R}^{+} \rightarrow \mathbb{R}^{+}$. From Lemma 10, we have that: for given $\epsilon>0$, if $x \theta(x) \nearrow \infty$, then there exists $x_{\epsilon}$ such that for all $x>x_{\epsilon}$,

$$
\int_{-\infty}^{x} e^{\theta(x) u} F(d u) \leq 1+c \theta^{1+\delta}(x)+e^{2 \alpha} \bar{F}\left(\frac{2 \alpha}{\theta(x)}\right)+e^{\theta(x) x} \bar{F}(x)(1+\epsilon),
$$

for some $\delta>0$. By definition of $\theta_{k}(b)$ in $(21)$, we have $\left(b+n_{k-1} \mu\right) \cdot \theta_{k}(b) \nearrow \infty$, either if $b$ or $k$ grows to infinity. Writing $\theta_{k}(b)$ as $\theta_{k}$, for values of $b$ and $k$ satisfying $b+n_{k-1} \mu>x_{\epsilon}$, we have,

$$
\begin{aligned}
\exp \left(\Lambda_{k}\left(\theta_{k}\right)\right) & \leq 1+c \theta_{k}^{1+\delta}+e^{2 \alpha} \bar{F}\left(\frac{2 \alpha}{\theta_{k}}\right)+e^{\theta_{k} \cdot\left(b+n_{k-1} \mu\right)} \bar{F}\left(b+n_{k-1} \mu\right)(1+\epsilon) \\
& \leq \exp \left(c \theta_{k}^{1+\delta}+e^{2 \alpha} \bar{F}\left(\frac{2 \alpha}{\theta_{k}}\right)+\frac{1}{n_{k}}(1+\epsilon)\right),
\end{aligned}
$$

because $1+x \leq e^{x}$ and $e^{\theta_{k} \cdot\left(b+n_{k-1} \mu\right)} \bar{F}\left(b+n_{k-1} \mu\right)=1 / n_{k}$. Then,

$$
\exp \left(n_{k} \Lambda_{k}\left(\theta_{k}\right)\right) \leq \exp \left(c n_{k} \theta_{k}^{1+\delta}+e^{2 \alpha} n_{k} \bar{F}\left(\frac{2 \alpha}{\theta_{k}}\right)+1+\epsilon\right) .
$$

Also see that,

$$
n_{k} \theta_{k}^{1+\delta}=\frac{n_{k}}{\left(b+n_{k-1} \mu\right)^{1+\delta}}\left(\log \left(\frac{1}{n_{k} \bar{F}\left(b+n_{k-1} \mu\right)}\right)\right)^{1+\delta}<\epsilon,
$$

if $b$ and $k$ are such that $\left(b+n_{k-1} \mu\right)$ is large enough. Similarly for given $\delta>0$, there exists $x_{\delta}$ such that if $b+n_{k-1} \mu>x_{\delta}$, then

$$
\begin{aligned}
\frac{\bar{F}\left(\frac{2 \alpha}{\theta_{k}}\right)}{\bar{F}\left(b+n_{k-1} \mu\right)} & =\frac{\bar{F}\left(\frac{2 \alpha\left(b+n_{k-1} \mu\right)}{-\log \left(n_{k} \bar{F}\left(b+n_{k-1} \mu\right)\right)}\right)}{\bar{F}\left(b+n_{k-1} \mu\right)} \\
& \leq\left(\frac{1}{2 \alpha} \log \left(\frac{1}{n_{k} \bar{F}\left(b+n_{k-1} \mu\right)}\right)\right)^{\alpha+\delta} .
\end{aligned}
$$


Then for values of $b$ and $k$ such that $\left(b+n_{k-1} \mu\right)$ is large enough,

$$
\begin{aligned}
n_{k} \bar{F}\left(\frac{2 \alpha}{\theta_{k}}\right) & \leq n_{k} \bar{F}\left(b+n_{k-1} \mu\right)\left(\frac{1}{2 \alpha} \log \left(\frac{1}{n_{k} \bar{F}\left(b+n_{k-1} \mu\right)}\right)\right)^{\alpha+\delta} \\
& =\frac{n_{k} L\left(b+n_{k-1} \mu\right)}{\left(b+n_{k-1} \mu\right)^{\alpha}}\left(\frac{1}{2 \alpha} \log \left(\frac{1}{n_{k} \bar{F}\left(b+n_{k-1} \mu\right)}\right)\right)^{\alpha+\delta}<\epsilon,
\end{aligned}
$$

because $\alpha>1$. Combining this with (62) and (63), for $b$ and $k$ such that $b+n_{k-1} \mu$ is sufficiently large,

$$
\exp \left(n_{k} \Lambda_{k}\left(\theta_{k}\right)\right) \leq \exp (1+3 \epsilon)
$$

thus establishing the claim.

Proof of Lemma 4. Since $n_{k}=r n_{k-1}$,

$$
\begin{aligned}
\sup _{k} \frac{n_{k} \bar{F}\left(b+n_{k-1} \mu\right)}{q_{k}(b)} & =\sup _{k} \frac{n_{k} \bar{F}\left(b+n_{k-1} \mu\right)}{\sum_{j=n_{k-1}+1}^{n_{k}} \bar{F}(b+j \mu)} \\
& \leq \frac{n_{k} \bar{F}\left(b+\frac{n_{k}}{r} \mu\right)}{\left(1-r^{-1}\right) n_{k} \bar{F}\left(b+n_{k} \mu\right)}<\infty
\end{aligned}
$$

because of (6).

Proof of Lemma 9. Since $\theta_{b} b \nearrow \infty$ and $\mathbb{E} X \neq 0$, similar to Lemma 10, we have:

$$
\begin{aligned}
\exp \left(\Lambda_{b}\left(\theta_{b}\right)\right) & \leq 1+\theta_{b} \mathbb{E} X+c \theta_{b}^{2}+\exp (2 \alpha) \bar{F}\left(\frac{2 \alpha}{\theta_{b}}\right)+\exp \left(\theta_{b} b\right) \bar{F}(b)(1+o(1)) \\
& =1-\theta_{b} \mu+c \theta_{b}^{2}+\exp (2 \alpha) \bar{F}\left(\frac{2 \alpha}{\theta_{b}}\right)+\frac{1}{b}(1+o(1)) .
\end{aligned}
$$

It follows from the definition of $\theta_{b}$ and a simple application of (6) that

$$
\frac{1}{b}=o\left(\theta_{b}\right) \text { and } \bar{F}\left(\frac{2 \alpha}{\theta_{b}}\right)=o\left(\theta_{b}\right), \text { as } b \nearrow \infty .
$$

Therefore,

$$
\exp \left(\Lambda_{b}\left(\theta_{b}\right)\right) \leq 1-\theta_{b} \mu(1+o(1))
$$

as $b \nearrow \infty$. Then

$$
\varlimsup_{b \rightarrow \infty} \sup _{n \geq 1} \exp \left(n \Lambda_{b}\left(\theta_{b}\right)\right) \leq \inf _{y} \sup _{n} \sup _{b>y}\left(1-\theta_{b} \mu(1+o(1))\right)^{n} \leq 1,
$$

which proves the claim. 


\section{REFERENCES}

[1] Adler, R. J., Feldman, R. E., And Taqqu, M. S., Eds. (1998). A practical guide to heavy tails. Birkhäuser Boston Inc., Boston, MA. Statistical techniques and applications. MR1652283

[2] Agarwal, A., Dey, S., And Juneja, S. (2013). Efficient simulation of large deviation events for sums of random vectors using saddle-point representations. Journal of Applied Probability 50, 3 (09), 703-720. http://dx.doi.org/10.1239/jap/1378401231.

[3] Asmussen, S. (1998). Subexponential asymptotics for stochastic processes: extremal behavior, stationary distributions and first passage probabilities. The Annals of Applied Probability 8, 2 (05), 354-374. http://dx.doi.org/10.1214/aoap/1028903531.

[4] Asmussen, S., Binswanger, K., And HøjgaArd, B. (2000). Rare events simulation for heavy-tailed distributions. Bernoulli 6, 2, 303-322. MR1748723

[5] Asmussen, S. And Glynn, P. (2007). Stochastic simulation: Algorithms and Analysis. Stochastic Modelling and Applied Probability, Vol. 57. Springer, New York. MR2331321

[6] Asmussen, S. And Kluppelberg, C. (1996). Large deviations results for subexponential tails, with applications to insurance risk. Stochastic Processes and their Applications 64, 1, $103-125$.

[7] Asmussen, S. And Kroese, D. P. (2006). Improved algorithms for rare event simulation with heavy tails. Adv. in Appl. Probab. 38, 2, 545-558. MR2264957

[8] Bassamboo, A., Juneja, S., and Zeevi, A. (2007). On the inefficiency of stateindependent importance sampling in the presence of heavy tails. Oper. Res. Lett. 35, 2, 251-260. MR2311409

[9] Bassamboo, A., Juneja, S., And Zeevi, A. J. (2008). Portfolio credit risk with extremal dependence: Asymptotic analysis and efficient simulation. Operations Research 56, 3, 593-606.

[10] Blanchet, J. and Glynn, P. (2008). Efficient rare-event simulation for the maximum of heavy-tailed random walks. Ann. Appl. Probab. 18, 4, 1351-1378. MR2434174

[11] Blanchet, J., Glynn, P., And Liu, J. (2007). Fluid heuristics, lyapunov bounds and efficient importance sampling for a heavy-tailed G/G/1 queue. Queueing Systems 57, 2$3,99-113$.

[12] Blanchet, J., Leder, K., And Glynn, P. (2009). Efficient simulation of light-tailed sums: an old-folk song sung to a faster new tune.... In Monte Carlo and quasi-Monte Carlo methods 2008. Springer, Berlin, 227-248. MR2743897

[13] Blanchet, J. and Liu, J. (2008). State-dependent importance sampling for regularly varying random walks. Adv. in Appl. Probab. 40, 4, 1104-1128. MR2488534

[14] Blanchet, J. AND Liu, J. (2012). Efficient simulation and conditional functional limit theorems for ruinous heavy-tailed random walks. Stochastic Processes and their Applications 122, 8, 2994 - 3031.

[15] Borovkov, A. A. And Borovkov, K. A. (2002). On probabilities of large deviations for random walks I. Regularly varying distribution tails. Theory of Probability and Its Applications 46, 2, 193-213. http://epubs.siam.org/doi/abs/10.1137/S0040585X97978877.

[16] Borovkov, A. A. And Borovkov, K. A. (2008). Asymptotic analysis of random walks. Encyclopedia of Mathematics and its Applications, Vol. 118. Cambridge University Press, Cambridge. MR2424161

[17] Borovkov, A. A. And Boxma, O. (2001). On large deviation probabilities of random walks with heavy tails. Preprint EURANDOM, Eindhoven. 
[18] Chan, H. P., Deng, S., And Lai, T.-L. (2012). Rare-event simulation of heavytailed random walks by sequential importance sampling and resampling. Advances in Applied Probability 44, 4 (12), 1173-1196. http://dx.doi.org/10.1239/aap/1354716593.

[19] Cline, D. B. And Hsing, T. (1991). Large deviation probabilities for sums and maxima of random variables with heavy or subexponential tails. Preprint, Texas A\&M University 501.

[20] Denisov, D. And Shneer, V. (2007). Local asymptotics of the cycle maximum of a heavy-tailed random walk. Advances in Applied Probability 39, 1 (03), 221-244. http://dx.doi.org/10.1239/aap/1175266476.

[21] Dieker, A. B. And Mandjes, M. (2005). On asymptotically efficient simulation of large deviation probabilities. Adv. in Appl. Probab. 37, 2, 539-552. MR2144566

[22] Dupuis, P., Leder, K., And Wang, H. (2007). Importance sampling for sums of random variables with regularly varying tails. ACM Trans. Model. Comput. Simul. 17, 3 (July).

[23] Dupuis, P. And Wang, H. (2004). Importance sampling, large deviations, and differential games. Stoch. Stoch. Rep. 76, 6, 481-508. MR2100018

[24] Embrechts, P., Klüppelberg, C., And Mikosch, T. (1997). Modelling extremal events. Applications of Mathematics (New York), Vol. 33. Springer-Verlag, Berlin. For insurance and finance. MR1458613

[25] Feller, W. (1971). An Introduction to Probability Theory and Its Applications Volume II. Wiley.

[26] Foss, S., Korshunov, D., And Zachary, S. (2011). An introduction to heavy-tailed and subexponential distributions. Springer Series in Operations Research and Financial Engineering. Springer, New York. MR2810144

[27] Glasserman, P. And Li, J. (2005). Importance sampling for portfolio credit risk. Management Science 51, 11, 1643-1656.

[28] Glynn, P. And Whitt, W. (1992). The asymptotic efficiency of simulation estimators. Oper. Res. 40, 3, 505-520. MR1180030

[29] Hammersley, J. M. And Handscomb, D. C. (1965). Monte Carlo methods. Methuen \& Co. Ltd., London. MR0223065

[30] JuneJA, S. (2007). Estimating tail probabilities of heavy tailed distributions with asymptotically zero relative error. Queueing Syst. 57, 2-3, 115-127.

[31] Juneja, S. and Shahabuddin, P. (2002). Simulating heavy tailed processes using delayed hazard rate twisting. ACM Trans. Model. Comput. Simul. 12, 2 (Apr.), 94-118.

[32] Juneja, S. And Shahabuddin, P. (2006). Rare event simulation techniques: An introduction and recent advances. Simulation, Handbooks in Operations Research and Management Science, 291-350.

[33] Korshunov, D. (1997). On distribution tail of the maximum of a random walk. Stochastic Processes and their Applications 72, 1, 97 - 103. http://www.sciencedirect.com/science/article/pii/S0304414997000604.

[34] Murthy, K. R. A. And JunejA, S. (2012). State-independent importance sampling for estimating large deviation probabilities in heavy-tailed random walks. In Performance Evaluation Methodologies and Tools (VALUETOOLS), 2012. 127 -135.

[35] PAReKh, S. AND Walrand, J. (1989). A quick simulation method for excessive backlogs in networks of queues. IEEE Trans. Automat. Control 34, 1, 54-66. MR970932

[36] Resnick, S. I. (1997). Heavy tail modeling and teletraffic data. Ann. Statist. 25, 5, 1805-1869. With discussion and a rejoinder by the author. MR1474072 
[37] Sadowsky, J. S. (1996). On Monte Carlo estimation of large deviations probabilities. Ann. Appl. Probab. 6, 2, 399-422. MR1398051

[38] Sadowsky, J. S. And Bucklew, J. A. (1990). On large deviations theory and asymptotically efficient Monte Carlo estimation. IEEE Trans. Inform. Theory 36, 3, 579-588. MR1053850

[39] Siegmund, D. (1976). Importance sampling in the Monte Carlo study of sequential tests. Ann. Statist. 4, 4, 673-684. MR0418369

[40] Veraverbeke, N. (1977). Asymptotic behaviour of Wiener-Hopf factors of a random walk. Stochastic Processes and their Applications 5, 1, 27 - 37.

School of Technology and Computer Science

Tata Institute of Fundamental Research

MUMBAi, INDIA

E-MAIL: kamurthy@tifr.res.in

Department of Industrial EngineEring juneja@tifr.res.in

AND Operations Research

Columbia University

IN THE CITY OF NEW YORK

E-MAIL: jose.blanchet@columbia.edu 